\title{
A convergent malignant phenotype in B-cell acute lymphoblastic leukemia involving the splicing factor SRRM1
}

\section{Adria Closa}

Australian National University

Marina Reixachs-Solé

Australian National University

Antonio Fuentes-Fayos

Maimonides Biomedical Research Institute of Cordoba https://orcid.org/0000-0003-2069-4823

Katharina Hayer

Children's Hospital of Philadelphia https://orcid.org/0000-0002-1463-3111

Juan Melero

Australian National University

Fabienne Adriaanse

Princess Maxima Center for Pediatric Oncology

\section{Romy Bos}

Princess Maxima Center for Pediatric Oncology

Manuel Torres-Diz

Children Hospital of Philadelphia https://orcid.org/0000-0002-5487-8113

\section{Stephen Hunger}

Children\&\#39;s Hospital of Philadelphia

\section{Kathryn Roberts}

St. Jude Children's Research Hospital https://orcid.org/0000-0001-7626-4043

\section{Charles Mullighan}

St. Jude Children's Research Hospital https://orcid.org/0000-0002-1871-1850

\section{Ronald Stam}

Princess Máxima Center https://orcid.org/0000-0003-4986-1656

\section{Andrei Thomas-Tikhonenko}

Children's Hospital of Philadelphia

\section{Justo Castaño}

IMIBIC-Univ of Cordoba https://orcid.org/0000-0002-3145-7287

\section{Raul Luque}

Maimonides Biomedical Research Institute of Cordoba (IMIBIC)

\section{Eduardo Eyras ( $\nabla$ eduardo.eyras@anu.edu.au )}




\section{Article}

\section{Keywords:}

Posted Date: January 12th, 2022

DOl: https://doi.org/10.21203/rs.3.rs-1168367/v1

License: (c) (1) This work is licensed under a Creative Commons Attribution 4.0 International License. Read Full License 


\section{A convergent malignant phenotype in B-cell acute lymphoblastic leukemia involving the splicing factor SRRM1}

Adria Closa ${ }^{1,2}$, Marina Reixachs-Solé, ${ }^{1,2}$, Antonio C. Fuentes-Fayos ${ }^{3,4,5}$, Katharina E. Hayer 6 , Juan Luis Melero $^{1,2}$, Fabienne R. S. Adriaanse ${ }^{7}$, Romy S. Bos ${ }^{7}$, Manuel Torres-Diz ${ }^{6}$, Stephen Hunger ${ }^{6}$, Kathryn G. Roberts ${ }^{8}$, Charles Mullighan ${ }^{8}$, Ronald W. Stam ${ }^{7}$, Andrei Thomas-Tikhonenko ${ }^{6,9}$, Justo P. Castaño ${ }^{3,4,5,10}$, Raúl M. Luque $3,4,5,10$, Eduardo Eyras ${ }^{1,2,11,12, *}$

${ }^{1}$ The John Curtin School of Medical Research, Australian National University, Canberra, Australia.

${ }^{2}$ EMBL Australia Partner Laboratory Network at the Australian National University, Canberra, Australia.

${ }^{3}$ Maimonides Biomedical Research Institute of Cordoba (IMIBIC), Cordoba, Spain.

${ }^{4}$ University of Cordoba (UCO), Cordoba, Spain.

${ }^{5}$ Reina Sofía University Hospital, Cordoba, Spain.

${ }^{6}$ Children's Hospital of Philadelphia, Philadelphia, USA.

${ }^{7}$ Princess Máxima Center for Pediatric Oncology, Utrecht, Netherlands

${ }^{8}$ St. Jude Children's Research Hospital, Memphis, USA.

${ }^{9}$ University of Pennsylvania School of Medicine, Philadelphia, USA.

${ }^{10}$ Centro de Investigación Biomédica en Red de Fisiopatología de la Obesidad y Nutrición, (CIBERobn), Cordoba, Spain.

${ }^{11}$ Catalan Institution for Research and Advanced Studies (ICREA), Barcelona, Spain.

${ }^{12}$ Hospital del Mar Medical Research Institute (IMIM), Barcelona, Spain.

*correspondence to: eduardo.eyras@anu.edu.au

\section{Abstract}

A significant proportion of B-cell acute lymphoblastic leukemia (B-ALL) patients remains with a dismal prognosis due to yet undetermined mechanisms. We performed a comprehensive multicohort analysis of gene fusions, gene expression, and RNA splicing alterations to uncover molecular signatures potentially linked to the observed poor outcome. We identified 84 fusions with significant allele frequency across patients. We identified an expression signature that predicts high risk independently of the gene fusion background. This signature includes the upregulation of the splicing factor SRRM1, which potentially impacts splicing events associated with poor outcomes through protein-protein interactions with other splicing factors. Experiments in B-ALL cell lines provided further evidence for the role of SRRM1 on cell survival, proliferation, and invasion. Our findings reveal a convergent mechanism of aberrant RNA processing that sustains a malignant phenotype independently of gene fusions and could complement current clinical strategies in B-ALL. 
B-cell acute lymphoblastic leukemia (B-ALL) is the most common form of childhood cancer worldwide and one of the leading causes of cancer-related deaths in children (Pui and Evans 2013). B-ALL presents a general lack of mutations in fundamental genes that drive the initial stages of the disease and are common in solid tumors and other leukemias, which could be therapeutically targeted (Gröbner et al. 2018). In contrast, B-ALL presents frequent chromosomal translocations that lead to the expression of gene fusions that are associated with marked differences in response to chemotherapy and survival. For instance, the frequent ETV6-RUNX1 and TCF3-PBX1 fusions have been associated with better prognosis (Boer and den Boer 2017), whereas BCR-ABL1, the highly frequent rearrangements of the gene KMT2A (KMT2A-r), and the less frequent TCF3-HLF are associated with poor prognosis (Fischer et al. 2015; Boer and den Boer 2017). Other rarer, less studied fusions remain with an uncertain prognosis.

The prevalence of gene-fusions has spurred multiple efforts to identify treatments that target them or their downstream effectors, albeit with limited success (Pikman and Stegmaier 2018). Many fusions involve transcription factors, which are difficult to target directly (Wang et al. 2017). Despite these challenges, combination chemotherapy and recent advances in Chimeric Antigen Receptor (CAR) T-cell therapy have led to a $90 \%$ increase in the 5 -year survival rate in children younger than 15 years and a 75\% increase for adolescents (15-19 years). However, infant B-ALL remains with a bad prognosis, especially for the KMT2A-r cases, which occur in about $80 \%$ of the infant patients during embryonic/fetal hematopoiesis (Bueno et al. 2011). A total of 135 different translocation partners have been identified for $K M T 2 A$, with the most frequent ones, accounting for $90 \%$ of all KMT2A-r cases, being members of transcriptional elongation complexes (Meyer et al. 2018). Furthermore, many of these fusions may occur at any age and are also frequent in acute myeloid leukemia (AML) (Meyer et al. 2018). Genome-sequencing studies of KMT2A-r B-ALL have confirmed a very low frequency of somatic mutations, suggesting that $K M T 2 A-r$ may not require additional alterations to induce transformation (Montes et al. 2011; Bursen et al. 2010). However, B-ALL cannot be recapitulated in pre-clinical models that only integrate the fusion, suggesting that additional alterations are necessary for the leukemogenesis (Bueno et al. 2012; Krivtsov and Armstrong 2007).

The rarity of many of the fusions in B-ALL and their link to functionally distinct pathways complicate their interpretation and the identification of effective therapies. On the other hand, there is 
increasing evidence for convergent molecular signatures that indicate similar disease progression patterns and common therapeutic vulnerabilities, despite presenting different genetic alterations. For instance, a BCR-ABL1-like B-ALL subtype was described that shows a gene expression profile and a therapeutic vulnerability similar to $B C R-A B L 1$ patients, despite not presenting the $B C R-A B L 1$ fusion (Tasian et al. 2017). For KMT2A-r fusions, the functional impacts of the different fusion gene-pairs have been linked to common downstream mechanisms of chromatin and transcription dysregulation (Krivtsov and Armstrong 2007). The occurrence of KMT2A-r across different ages and lineages and their general association with poor prognosis suggests that a convergent phenotype might be potentially identified in B-ALL in association with poor prognosis. We hypothesized that this phenotype might be captured in the transcriptome and present in high-risk B-ALL tumors independently of the fusion background.

Here we describe a comprehensive multi-cohort age-agnostic characterization of B-ALL transcriptomics signatures of high risk. We identified a predictive signature of high risk independent of the fusion background. This signature is mainly composed of ribosome biogenesis and RNA processing regulators, including the splicing factor SRRM1. We found an alternative splicing program associated with the high-risk signature potentially mediated by splicing factors interacting with SRRM1. Furthermore, in vitro experiments in B-ALL cell lines provide further evidence for the functional role of SRRM1 in cell survival, proliferation, and invasion. Our results provide a new layer of molecular variation that has remained undetected so far and represents a potential source of novel prognostic markers and therapeutic strategies in B-ALL.

\section{Results}

\section{RNA sequencing identifies novel gene fusions in B-ALL}

We collected RNA sequencing (RNA-seq) from five different B-ALL studies (Lilljebjörn et al. 2016; Black et al. 2018; Agraz-Doblas et al. 2019; Ma et al. 2015; Andersson et al. 2015), corresponding to 428 patient samples obtained at diagnosis, plus 82 samples from relapsed cases, 55 of them paired (Supp. Table 1 and Data file 1). Patients' age distribution peaked around one year old, with most cases being classified as infant or young children (Fig. 1a). We applied a comprehensive pipeline to these RNA-seq datasets to study gene fusions, expression, and RNA splicing (Supp. Fig. 1) (Methods). 
To identify fusions likely to be associated with the B-ALL phenotype, we removed fusion candidates that were also detected in non-cancer tissues and normal hematopoietic cells, as well as potential artifacts. We kept all fusion candidates reported before in the studied cohorts or involving genes with acute leukemia mutations in COSMIC (Tate et al. 2019). Additionally, only candidates appearing in 5 or more samples and across different projects were considered. Starting from 1,825 unique candidate fusions, these filters resulted in 158 unique high-confidence fusions (Supp. Fig. 2).

To prioritize the relevance of the identified fusions, we further calculated a fusion allele frequency (FAF)(Haas et al. 2019), defined as the proportion of the gene expression corresponding to a fusion averaged over the two genes participating in the fusion, and which represent a proxy for the fusion clonality. The most frequent fusions in the studied cohorts showed larger FAF values, and the distribution of FAF values for all fusions had peaks near 1, 0.5, and 0 (Supp. Fig. 3). When we considered the FAF values for each gene in the fusion separately, in most cases, the genes in the fusion showed significantly different distributions (Supp. Fig. 3). This indicates that, in general, the expression of both genes in the fusion is affected differently with respect to the wild-type gene. We thus considered only those fusions with a median FAF of at least 0.1 and having none of its genes with a median individual FAF below 0.1 . These analyses resulted in our final list of 84 different fusions (Data file 2).

Using these fusions, all 5 analyzed cohorts presented a similar distribution of fusions per patient (Supp. Fig. 4). Our analyses of the RNA-seq data recovered $81.35 \%$ of the most frequent fusions detected in the same samples with independent experimental methods (Supp. Fig. 5). Moreover, we found an overrepresentation of some of the detected fusions in specific age groups (Fig. 1b). We recovered the known enrichment of KMT2A-r in infant cases and ETV6-r in children and young adults (1-18 years). Fusion groups such as BCR-r and P2RY8-r presented a bimodal or extended age distribution, including infant and child. Similarly, PAX5- $r$ appeared in infants and at the upper extreme of the childhood cases (Supp. Fig. 6). However, age distributions and fusion frequencies may not be in all cases representative of the distribution in the population as there have been sample collection biases in each cohort.

We predicted known B-ALL fusions in 43 patients that did not have any fusion annotated in their study and identified the fusion partner in 7 cases that were only annotated as KMT2A-r without 
their specific partner (Data file 2). Moreover, we identified fusions that had only been reported before in solid tumors, such as ST3GAL1-NDRG1, P2RY8-AKAP17A, and SS18L1-ADRM1, and various fusions that had not been previously described in B-ALL or any other cancer, including GSE1-SLC7A5 and CBFA2T3-PIEZO1 (Fig. 1c) (Data file 2). Overall, high-confidence fusions were detected for 70\% of the samples at diagnosis. Grouping the fusions by the most frequent gene in the fusion pairs, we could identify six major fusion groups: KMT2A-r (20\%), ETV6-r (16\%), ST3GAL1-r (8\%), P2RY8-r (8\%), TCF3-r (8\%), and TTYH3-r (7\%) (Fig. 1b). In the cohorts analyzed, we also found PAX5-r (3\%), ZNF384$r(3 \%), B R C-r(2 \%), R U N X 1-r(2 \%)$, and GSE1-r (2\%), as well as a group of low-frequency fusions that appeared in $18 \%$ of the patients (Fig. 1b). The calculated fusions in diagnostic samples showed a clear pattern of mutual exclusions and a frequent co-occurrence of the inverse fusion for KMT2A-r, ETV6-r, PAX5-r, and BCR-r, but not for other fusions (Fig. 1c).

a

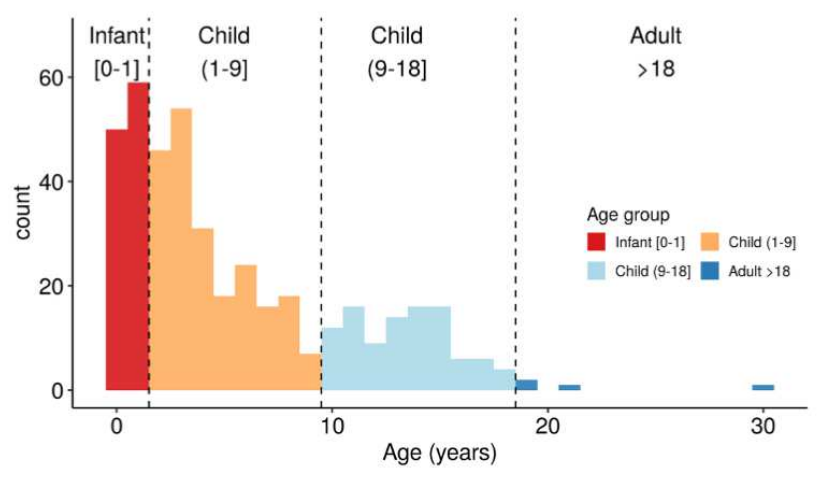

b

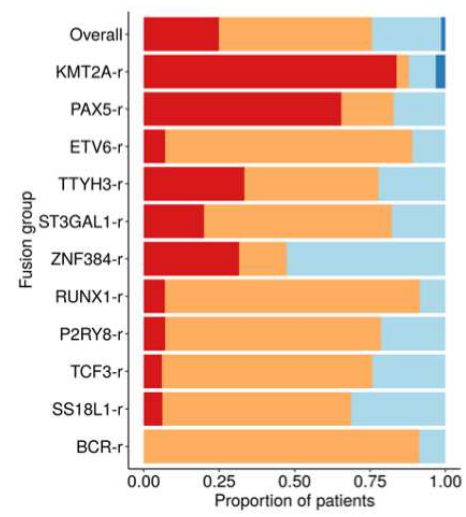

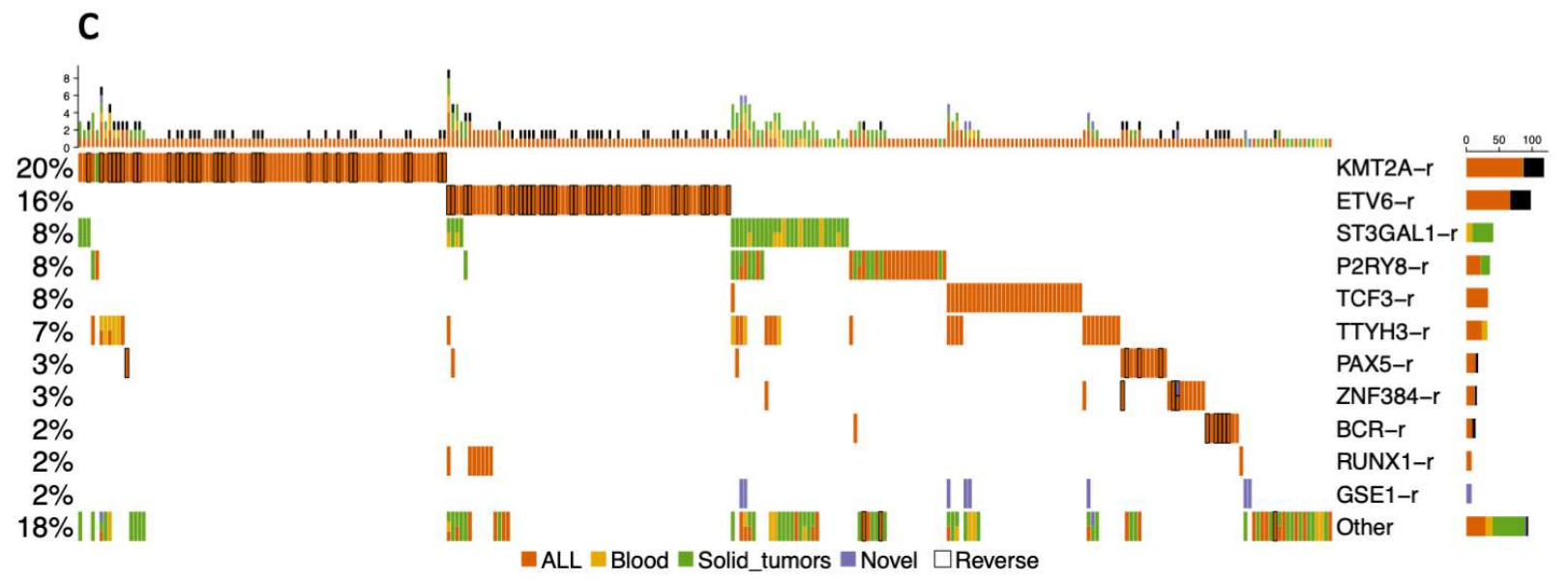

Figure 1. Multicohort identification of gene fusions in B-ALL. (a) Age distribution of the B-ALL patients studied. (b) The proportion of the gene fusion groups in each age group from (a). (c) Fusion oncoprint. The plot shows the most frequent fusions (rows) detected in patients (columns). Fusions are grouped by the most frequent gene in the fusion gene pairs. The bar plot above shows the number of fusions detected per patient. The specific gene fusion pairs in each patient are given in (Supp. Table 2). A 
black border line indicates that the reverse fusion was identified in that patient. Cell colors indicate whether the fusions were detected before in B-ALL (red), in a blood cancer (orange), in a solid tumor (green), or whether it was not reported in any cancer before (purple). A patient with two different fusions detected in the same gene is depicted with two colors, and they were only kept if they had been previously reported in a tumor and occurred in at least five patients.

\section{Different gene fusions impact similar functional pathways}

We calculated the breakpoints for each of the fusions detected from RNA-seq reads, either as the exon position where the fusion breakpoint was found or using the boundaries of the exons flanking the intron where the breakpoint was assumed to fall. Fusion genes presented multiple breakpoints (Fig. 2a) (Supp. Fig. 7). Moreover, these breakpoints appeared in positions that potentially disrupted the protein domain content. This raised whether different breakpoints in the same or different fusion genes may lead to similar functional impacts. To determine this, we calculated the domains that are maintained or lost in each fusion gene according to the identified breakpoints. In terms of the specific domains kept or lost in a fusion, we observed little overlap between fusions (Supp. Fig. 8). However, when we grouped the protein domains according to their functional ontologies, multiple similarities appeared, such as the loss of DNA-binding domains in KMT2A-r and ETV6-r and the loss of signal transduction domains in P2RY8-r and BCR-r (Fig. 2b).

\section{Common patterns of gene expression across diverse gene fusion backgrounds}

To further characterize the cellular processes associated with the identified fusion groups, we calculated the differential expression patterns among patient groups. Despite the heterogeneity of samples used in the comparison, there were many significant expression changes associated with KMT2A-r, ETV6-r, ST3GAL1-r, ZNF384-r, and TCF3-r in comparison with the other fusion groups and with patients without fusions (Supp. Fig. 9) (Data file 3). These patterns included the HOXA overexpression characteristic of KMT2A-r patients (Supp. Fig. 10) (Agraz-Doblas et al. 2019). Interestingly, the differentially expressed genes associated with each fusion group did not generally overlap (Supp. Fig. 11), except for the KMT2A-r and ETV6-r groups, which showed opposite expression patterns (Supp. Fig. 12). This suggested that the functional alterations specific to KMT2A$r$ are reversed in ETV6-r tumors. 
a

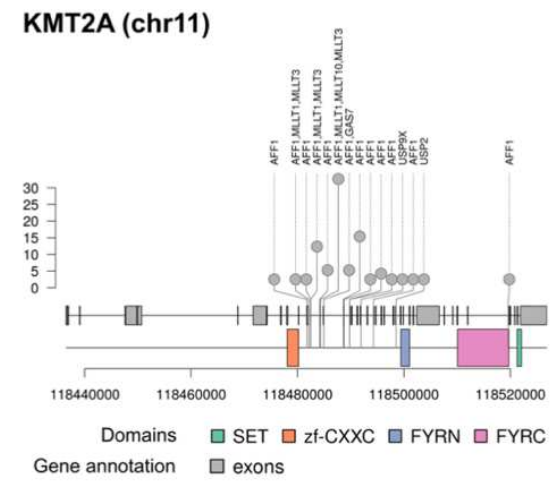

ETV6 (chr12)

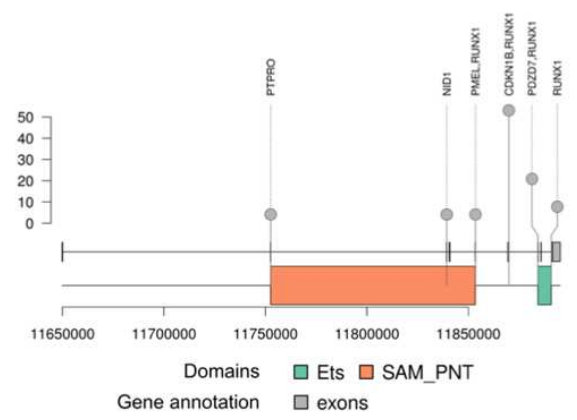

b

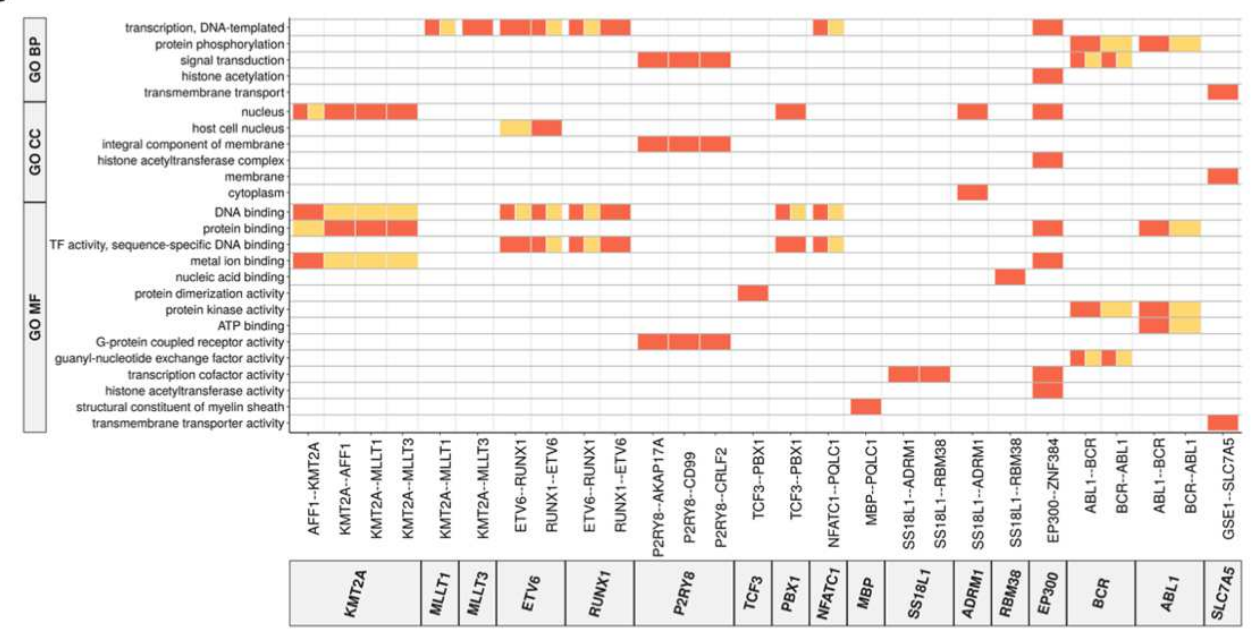

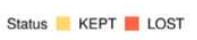

Figure 2. Functional impact assessment of gene fusions. (a) Identified breakpoints in the fusions involving KMT2A (left panel) and ETV6 (right panel). Along the genomic locus ( $\mathrm{x}$-axis), we indicate the detected breakpoint's position, defined by an exonic position if the breakpoint occurred inside an exon, or by the last base of the last exon included in the fusion otherwise. The plot also shows the span of the functional domains in genomic coordinates. (b) Domains kept (orange) and lost (red) through gene fusions (x-axis) labeled according to gene ontologies (GO) (y-axis): Biological Process (BP), Cellular Compartment (CC), and Molecular Function (MF). The specific functional domains kept or lost in these fusions are given in Supplementary Figure 8.

To investigate this possibility, we studied the pathways enriched or depleted in each fusion group. Each group tended to cluster independently, except for ST3GAL1-r, PQLC1-r, and TTYH3-r, which presented similar pathway enrichments and P2RY8-r, RUNX1-r, and PAX5-r, which were similar to the cases with no fusions (Supp. Fig. 13). Interestingly, MYC targets were upregulated in KMT2A-r patients but depleted in ETV6-r (Supp. Fig. 13). Additionally, transforming growth factor beta (TGF- 
b) signaling, which antagonizes MYC (Ning et al. 2019), was depleted in KMT2A-r patients. Furthermore, MYC expression was higher in $K M T 2 A-r$ patients relative to the other patient groups and normal fetal-liver B-cells (Supp. Fig. 14), whereas ETV6-r patients showed MYC expression below normal fetal liver B-cells (Supp. Fig. 14). This suggested a gene expression pattern linked to MYC, associated with KMT2A-r, and reversed in ETV6-r.

\section{A gene expression signature of high-risk B-ALL independent of the gene fusion background}

B-ALL cases with KMT2A-r are generally considered to have a poor prognosis, whereas ETV6-r are deemed to be of good prognosis (Boer and den Boer 2017). We thus reasoned that the observed opposite expression patterns might be linked to these risk phenotypes. To investigate this possibility, we calculated the genes differentially expressed between patients harboring only KMT2A-r or ETV6-r, without any other detected fusion (87 KMT2A-r and 68 ETV6-r). Gene set enrichment analysis on the 1405 differentially expressed genes identified (FDR $<0.01$ and $|\operatorname{logFC}|$ >1) (Fig. 3a) (Data file 4) revealed two main pathways, one associated with immune response and another related to MYC, translation regulation, and ribosome biogenesis (Fig. 3b). Moreover, genes overexpressed in KMT2A-r patients were strongly enriched in MYC targets, ribosome biogenesis, and RNA processing, including splicing and translation regulation (Supp. Fig. 13). RNA processing is tightly controlled during normal hematopoiesis and is commonly dysregulated in the hematological malignancies (Signer et al. 2014; Hodson et al. 2019; Koh et al. 2015). We thus hypothesized that genes related to these processes may separate patients according to their risk of relapse. Feature selection was conducted to extract a list of 39 genes from the target pathways showing overexpression in KMT2A-r and patients with relapse risk. RBM24 and RNU6-1 were discarded from these genes as they showed high variability across the studied cohorts, resulting in a final signature of 37 genes (Fig 3c) (Supp. Fig. 15).

We trained and tested a predictive model for risk using the 133 patients from TARGET that had clinical follow-up information (Data file 1), using as end-point the event-free survival. Using a random forest leave-one-out cross-validation combined with a cox-regression, we obtained a significant separation of patients according to the risk of relapse (log-rank test $p$-value $<0.0001)$ (Fig. 3d) and overall accuracy of 0.72, measured as the area under the receiver operating characteristic (ROC) curve (AUC) (Fig. 3e). Importantly, even though the genes in our model were 
selected from the comparison of KMT2A-r and ETV6-r patients, only $11 \%$ of the 133 patients had KMT2A-r or ETV-6 fusions. Based on the observed values of sensitivity, specificity, and accuracy (Supp. Fig. 16a), we chose the decision boundary at score 0.7 ( $>=0.7$ for high risk and $<0.7$ for low risk). However, the same accuracy values were maintained with score thresholds between 0.5 and 0.7 (Supp Fig. 16a).

To further evaluate our predictive signature, we trained a single model that included all 133 TARGET samples used above (Supp. Fig. 16b). We applied this model to all other B-ALL samples and normal samples from GTEX for blood and spleen. Importantly, samples obtained at relapse, which were not included in any of the analyses above, showed a distribution similar to the high-risk samples and higher than the normal samples (Fig. 3f), which had also not been used before. The threshold applied to the score separated TARGET patients with and without relapse and detected other highrisk patients from the other cohorts (Fig. 3g) (Supp. Fig. 16c). Our predictions showed that patients with fusions KMT2A-AFF1, AFF1-KMT2A, and TCF3-PBX1 were more frequently in the high-risk group. In contrast, patients with ETV6-RUNX1, RUNX1-ETV6, and ABL1-BCR were more often classified as low risk, including the ability to stratify patients with higher risk inside every group of fusion (Fig. 3g) (Supp. Fig. 16c) (Data file 5).

We further applied our model to an independent cohort of 188 B-ALL patients (Jeha et al. 2021). Since the gene expression values for this dataset were only available in terms of regularized log (rlog) values, we rebuilt our model using the rlog expression values calculated from the TARGET samples. Using a leave-one-out cross-validation, this model maintained the same classification power as the previous model built with logCPM values (Supp. Fig. 17a), and the scores given by both models showed a high correlation ( $R=1, p$-value<2.2e-16) (Supp. Fig. 17b). We then used the clinical classification of patients published by the independent study into three different risk groups (high, standard, and low) (Jeha et al. 2021). Our model separated these three groups into significantly different score distributions, with the high-risk group showing the highest scores and the low-risk group showing the lowest signature scores (Supp. Fig. 17c). These analyses provide strong support for the ability of our model to predict the potential for relapse in B-ALL independently of the genefusion background. To make this model readily available to evaluate the risk on new sets of patients from expression data and to explore the features of the TARGET cohort, we integrated the predictive model on an interactive web resource available at https://github.com/comprna/risk model app. 
a

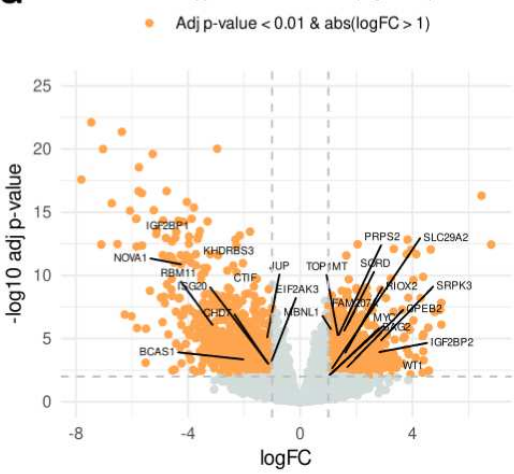

b

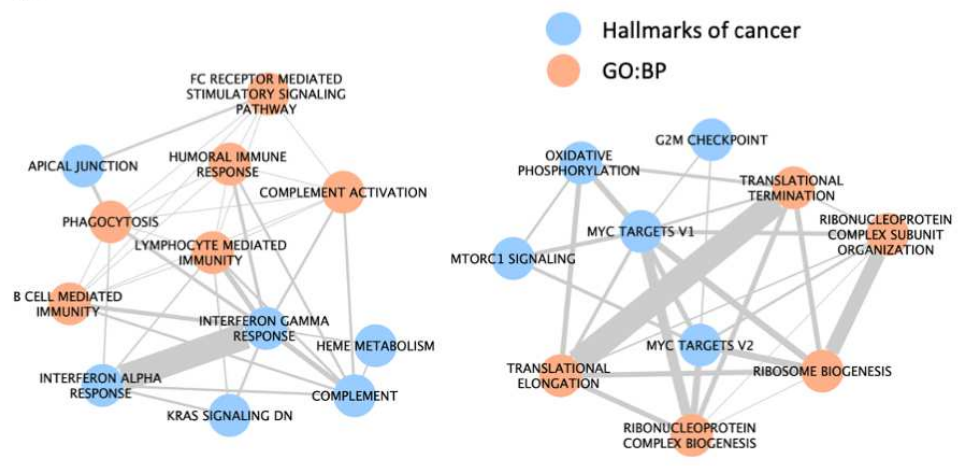

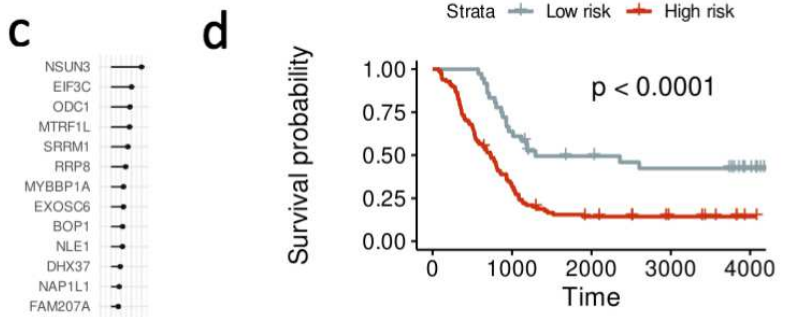

Number at risk

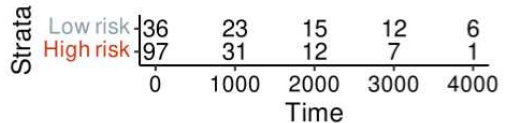

f

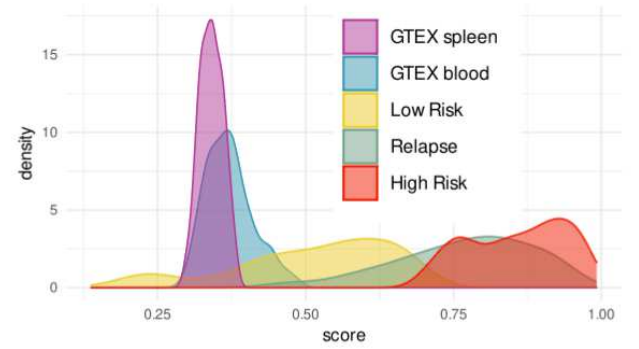

e

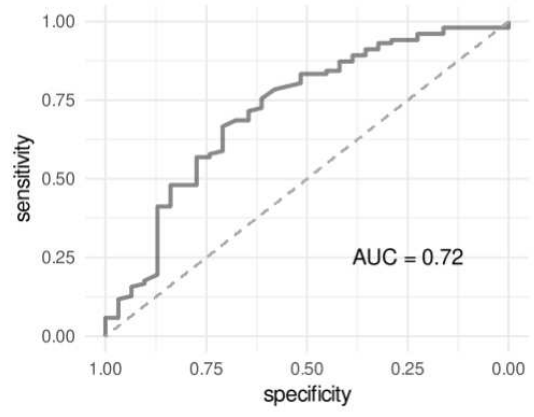

g

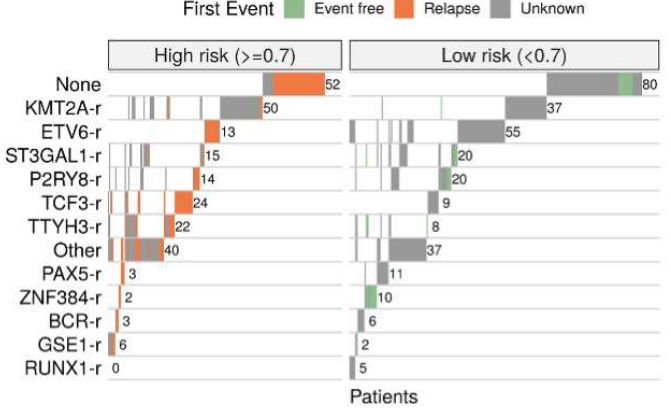

Figure 3. Gene expression signature of high risk. (a) Differential gene expression between a subset of patients with only KMT2A-r or only ETV6-r. The volcano plot shows for each gene the log2(fold-change) (xaxis) and the - $\log 10$ (corrected p-value) (y-axis). We indicate the genes involved in RNA processing, RNA translation, and Ribosome biogenesis. (b) Enriched Cancer Hallmarks and Biological Process Gene Ontologies (GO:BP) of the genes significantly up or down-regulated in the comparison in (a). (c) Ranking of the 37 genes of the predictive model of high risk according to their relevance in the leave-one-out test ( $x$ axis). Relevance is defined as the median of the accuracy per gene. (d) Kaplan-Meyer plot of the patients separated as high risk (red) (risk score $>=0.7$ ) or low risk (grey) (risk score $<0.7$ ) in a leave-one-out test. (e) Average receiving operating characteristic (ROC) curve and area under the ROC curve (AUC) from the leave-one-out test for classifying patients into low and high risk. The ROC curve shows the specificity (xaxis) and sensitivity (y-axis) for the entire range of possible model score threshold values. Sensitivity is defined as the proportion of high-risk cases that are correctly predicted. In contrast, specificity is defined as the proportion of low-risk cases correctly predicted as low risk. (f) Risk score distribution for various sample 
groups: diagnostic samples predicted as high risk, diagnostic samples predicted as low risk, samples obtained at relapse, and blood and spleen samples from GTEX. (g) Classification of all the B-ALL diagnostic samples from each fusion subgroup ( $y$-axis) into high (left) or low (right) risk according to our risk score. Patients with a clinical record indicating that they had relapsed are indicated in orange, whereas patients annotated with no relapse are indicated in green (Event free). Patients with no clinical information are indicated in grey (unknown).

\section{A splicing program associated with high-risk B-ALL}

Our high-risk signature included several splicing factors (SFs) and genes encoding for RNA binding proteins (RBPS), such as SRRM1, IGF2BP2, and MBNL1, which suggested a pattern of differential RNA processing linked to high risk. Analysis of differential RNA processing events between patients predicted as high and low risk identified a total of 422 events, with 342 of them affecting coding transcripts and showing enrichment of alternative first (AF) and skipping exons (SE) (Fig. 4a) (Data file 6). Interestingly, events that differentiate the high and low-risk patients had a small overlap with those that differentiate between patients with KMT2A-r and ETV6-r. The genes affected by differential included events also showed a small overlap (Supplementary Fig. 18) (Data file 7). Moreover, the identified significant events can separate between high and low-risk patients (Fig. 4b) (Supplementary Fig. 19).

To evaluate the SFs/RBPs potentially associated with these RNA processing changes, we performed a motif enrichment analysis. Specifically, we identified motifs for RBPs that were part of our highrisk signature: SAMD4A in $A 3$ and $A L$ events, $R B M 24$ in all the different types of events, $P C B P 3$ in $A L$ and SE events, PABPC4 in AL, and MBNL1 in A3, AL, RI and SE events. We also recovered motifs for other RBPs, including the SR protein genes SRSF1, SRSF2, SRSF7, and SRSF9. Interestingly, these SR proteins and other SFs/RBPs with motifs enriched in events showed protein-protein interactions with signature members (Fig. 4c) (Supp. Fig. 20). Most event types were enriched in motifs RBM28, an interactor of 5 of the proteins encoded by genes of our high-risk signature: RRP8, RRP15, UTP20, $B O P 1$, and $D H X 37$ (Fig. 4d). Similarly, we found an enrichment of motifs for RBFOX1, which interacts with RBM24, also part of the signature (Fig. 4d). Remarkably, there were 9 RBPs with motif enrichment in the events changing with the risk that had protein-protein interactions with the splicing factor SRRM1 (Fig. 4d). SRRM1 has one of the largest predictive values in our high-risk 
signature (Fig. 3c) and is the splicing factor with the highest correlation with the risk score (Pearson $r=0.50 \mathrm{p}$-value $=8.55 \mathrm{e}-29)$. These analyses suggest that a high-risk phenotype may be associated with a change in the expression of multiple SFs and RBPs that impact RNA processing, with a potentially prominent role for SRRM1.

\section{a}

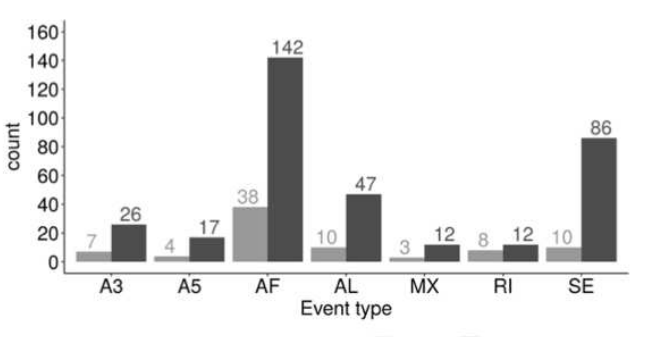

Impact in coding region 1 FALSE $\mathbf{D}$ TRUE

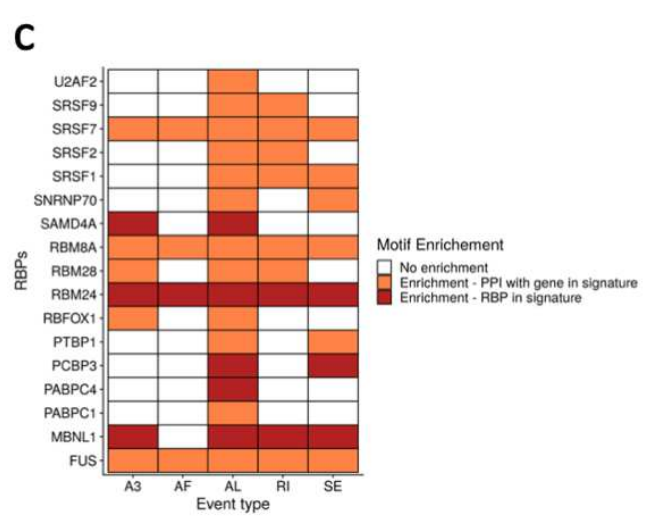

b

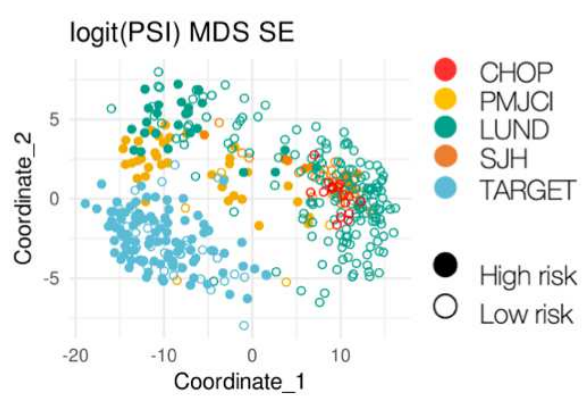

d

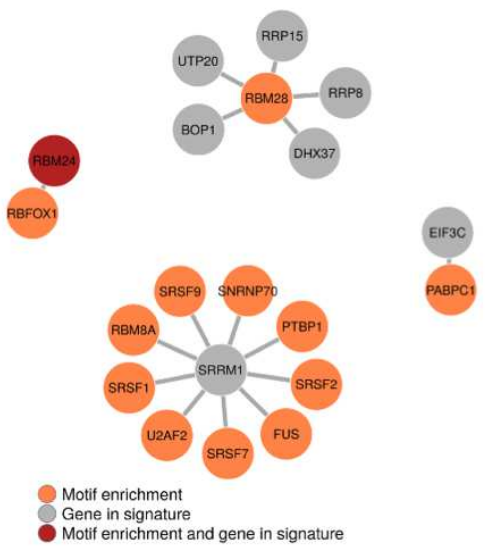

Figure 4. Splicing signature associated with high-risk. (a) Identified significant splicing changes between high and low-risk patients. The counts are separated according to whether the splicing change impacts a coding region (black) or not (grey), and by event type: alternative $3^{\prime}$ (A3) and 5' (A5) splice site, alternative first (AF), and alternative last (AL) exon, mutually exclusive exons (MX), retained intron (RI) and skipping exon (SE). (b) Multidimensional scaling (MDS) plot of the analyzed samples using only the significative SE events. The color indicates the patient cohort, and the full (empty) circle indicates if the sample is predicted to be of high (low) risk. Other event types are shown in Supplementary Fig. 19. (c) Enrichment (z-score > 1.5) of binding motifs for RNA binding proteins (RBP) ( $y$-axis) in each event type (x-axis). The plot indicates whether the RBP is part of the high-risk signature (red) or whether it has a reported protein-protein interaction (PPI) with a gene from the signature (orange). (d) Protein-protein interaction networks for the RBPs in the predictive signature and/or with binding motifs enriched in the events differentially spliced between high and low-risk patients. The color indicates whether the RBP motif was enriched (orange), whether the RBP was part of the high-risk signature (gray), or both (red). 


\section{SRRM1, a potential master regulator of high-risk associated splicing}

SRRM1 codes for a Serine-Arginine rich factor that associates with splicing complexes and affects splicing through interactions with SR proteins (Blencowe et al. 1998) (Fig. 5a). Patient samples classified as high risk and relapse samples showed higher SRRM1 expression than low-risk samples and normal samples from spleen and blood (Fig. 5b). Moreover, SRRM1 expression correlated with the inclusion of differentially spliced events between high and low-risk patients across all samples when those events contained binding motifs for the RBPs interacting with SRRM1 (Supp. Fig. 21). Interestingly such correlation was not always observed for the RBPs, indicating that in some cases, the regulation may be directly linked to SRRM1.

To investigate the possible mechanisms linking SRRM1 with high risk, we calculated the subset of splicing events associated with risk that was also significantly associated with risk within each fusion group. Most of these events co-occurred with patients with KMT2A-r, ETV6-r, TCF3-r, as well as patients with no fusions (Fig. 5c, lower panel) (Supp. Fig. 22); and presented binding motifs for RBPs interacting with SRRM1 (Fig. 5c, middle panel). Moreover, the genes harboring those events were significantly associated with cancer-related pathways, such as MYC targets, DNA repair, and the p53 pathway (Fig 5c, upper panel). One of these genes was EIF4H, a translation initiation factor that is key for translational control. Overexpression of EIF4H has been associated before with cell proliferation and increased chemoresistance in the lung cancer (Vaysse et al. 2015). Our analyses indicated that one of the EIF4H isoforms (ENST00000265753.12) decreased expression in the lowrisk group, while a second isoform (ENST000000353999.6) had stable expression across all patients (Supp. Fig. 23).

To further understand the functional transformations associated with SRRM1 expression, we analyzed multiple samples from progenitor and mature B-cells. SRRM1 was significantly downregulated in mature B-cells compared with B-cells progenitors (Fig. 5d). Consistent with this, the risk score was significantly higher (0.50-0.73) in undifferentiated B-cells compared to differentiated B-cells (0.38-0.46) ( $p$-value < 0.001) (Supp. Fig. 24) (Data file 5).

To evaluate whether SRRM1 could play a role in the splicing changes across B-cell differentiation, we calculated the differentially spliced events and performed motif enrichment between 
progenitors and mature B-cells. Strikingly, differentially spliced events between progenitors and mature B-cells contained motifs for the same SRRM1-interacting RBPs and splicing factors that we observed for leukemia patients (Fig 5e). Additionally, the genes with differentially spliced events were enriched in the same pathways obtained in the comparison between high and low risk patients, which were different from the pathways enriched in genes with splicing differences between KMT2A-r and ETV6-r (Supp. Fig 25) (Data file 8). Interestingly, the expression level of the KMT2A-AFF1 and ETV6-RUNX1 fusions, the most abundant of the KMT2A-r and ETV6-r groups, did not show any correlation with the risk score or with the SRRM1 expression levels (Supp. Fig. 26). These results lead us to think that a molecular signature possibly driven by SRRM 1 and associated with an undifferentiated phenotype is associated with a bad prognosis in B-ALL and has a better predictive value than the differences explained by the fusion background.

a

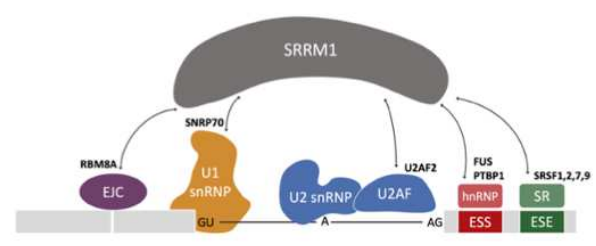

C

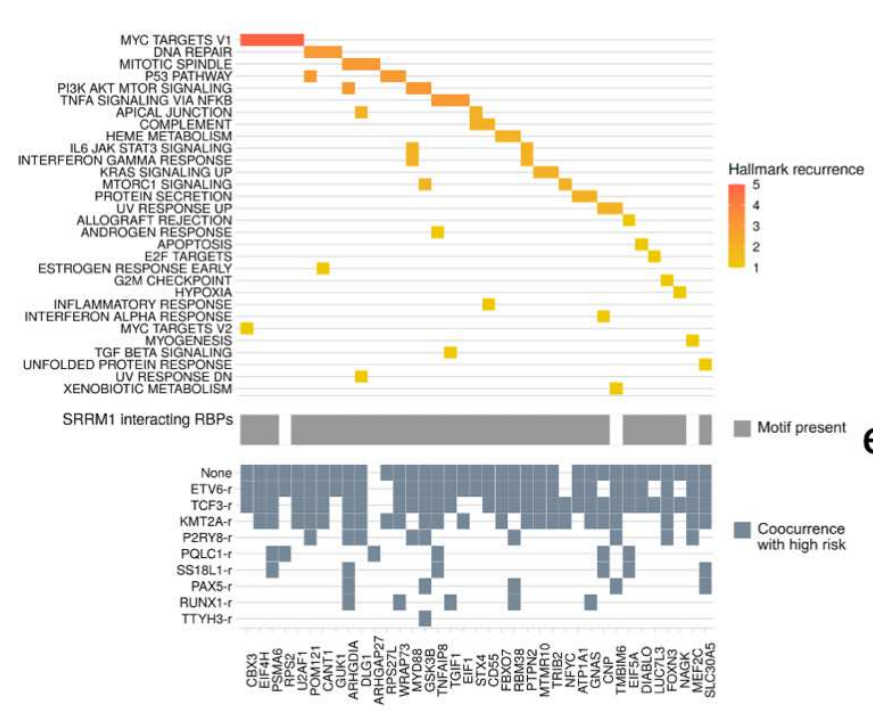

b
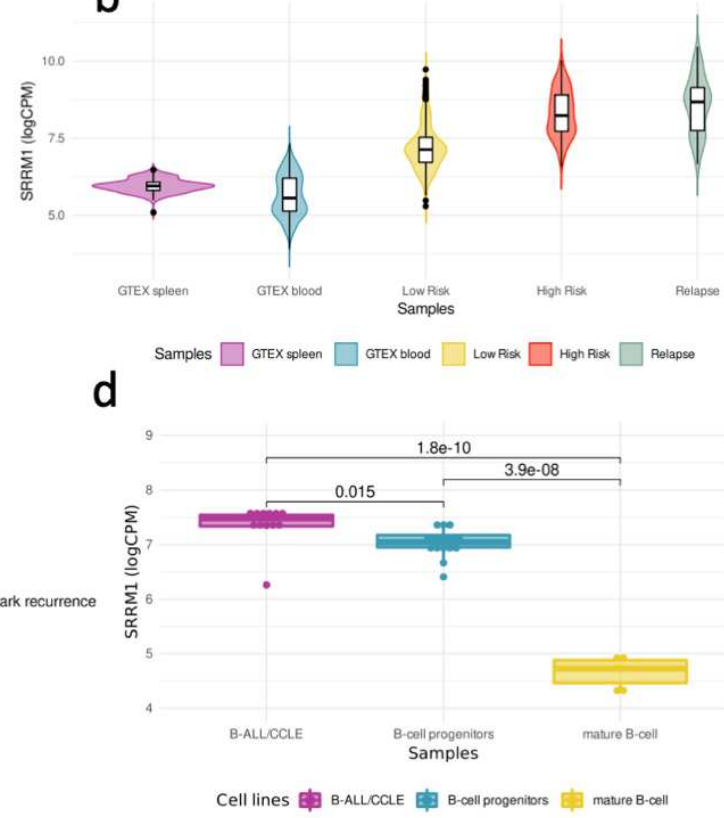

e

Motif enrichment and PPI with SRRM1

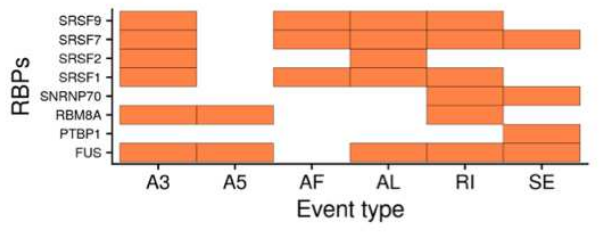

Figure 5. SRRM1 as a potential major driver of high-risk B-ALL. (a) SRRM1 is a known interactor of multiple splicing factors and RNA binding proteins (RBPs). (b) Distribution of expression values for SRRM1 in $\log 10$ counts per million (logCPM) units (y-axis) in various sample groups: B-ALL diagnostic samples predicted to be high risk (score $>0.7$ ), B-ALL diagnostic samples predicted to be low risk (score $<0.7$ ), B-ALL relapse samples, 
and blood and spleen samples from GTEX. (c) Upper panel shows cancer hallmarks associated with genes with differentially spliced events that co-occur with high risk in at least one fusion group, colored by its recurrence. In grey, the middle panel indicates the presence of binding motifs for any of the RBPs that interact with SRRM1. Lower panel highlights in blue the fusion groups in which the differentially spliced events cooccur with high risk. (d) Normalized log2CPM expression of SRRM1 in B-ALL cell lines from the cancer cell line encyclopedia (CCLE), B-cell progenitors, and a set of GM12878 biological replicates. P-values were obtained from a t-test mean comparison. (e) RBPs and splicing factors with associated motifs enriched in events differentially spliced between mature B-cells and B-cell progenitors.

\section{Impact of SRRM1 silencing in key functional parameters of human leukemia cells}

To uncover the impact of SRRM1 in key functional tumor features, we determined proliferation, apoptosis, and invasion rate, after silencing SRRM1 in six human B-ALL leukemia cell line models bearing distinct functional and phenotypic features: SEM, MHH-CALL-3, KOPN-8, NALM-19, REH, and SUP-B15. Silencing of SRRM1 expression was successful in all cell models (Fig. 6a) and resulted in a significant decrease in proliferation rate in a time-dependent and cell line-dependent manner (Fig. 6b). Furthermore, the use of the capase3/7 assay revealed that SRRM1 silencing significantly induced apoptosis in all human leukemia cells (Fig. 6c). Moreover, the trans-well assay used to evaluate the invasion capacity revealed that SRRM1 silencing could potentially impair the capacity of these cells to invade surrounding tissues in all cell lines tested (Fig. 6d). SRRM1 expression analysis revealed that the cell lines had different basal expression patterns (from high to low levels: KOPN8>SEM $>$ REH $>$ MHH-CALL-3>NALM-19>SUP-B15), with differences in the basal proliferation as well

(Fig. 6e). Furthermore, the proliferation rate with the siRNA at $48 \mathrm{~h}$ was inversely correlated with the SRRM1 basal expression and the silencing effectiveness (Fig. 6e), arguing in favor of the tight association of SRRM1 expression with this essential cell function. 
a

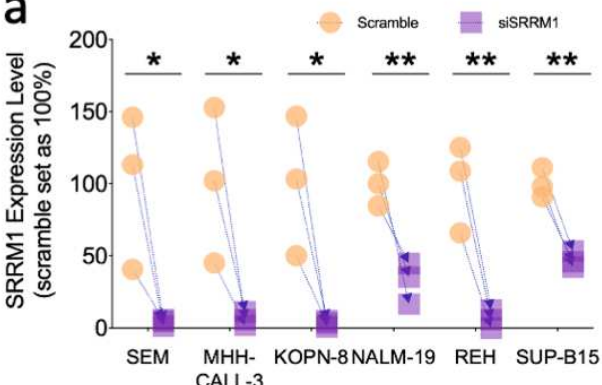

C Luminescence (RLU) Background Substracted (\% apoptosis induction vs. Scramble siRNA)

0

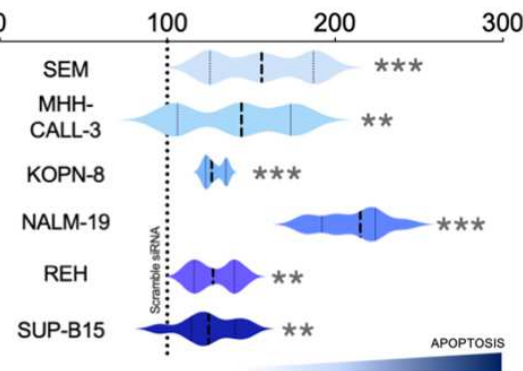

d

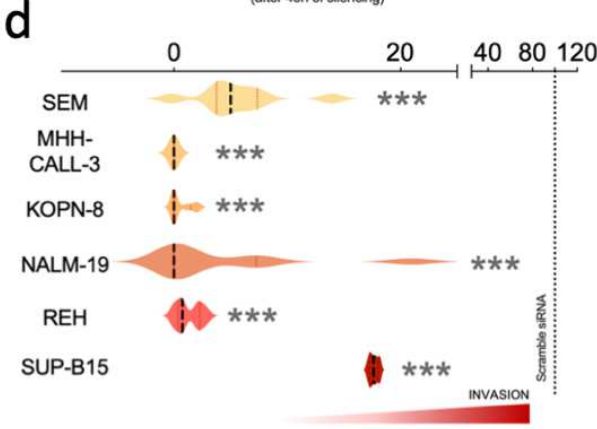

b

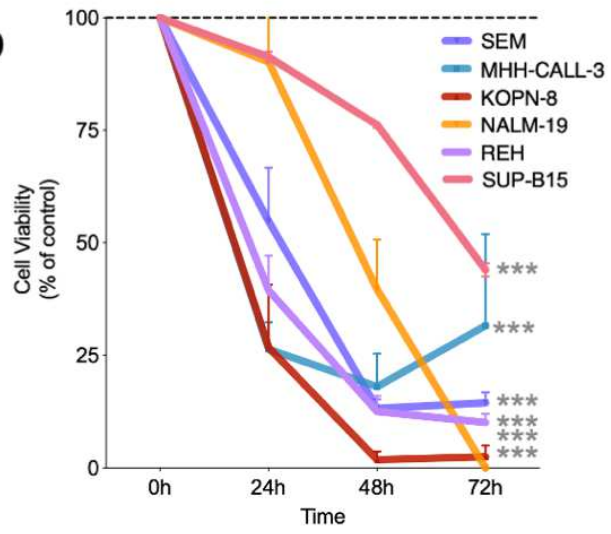

e
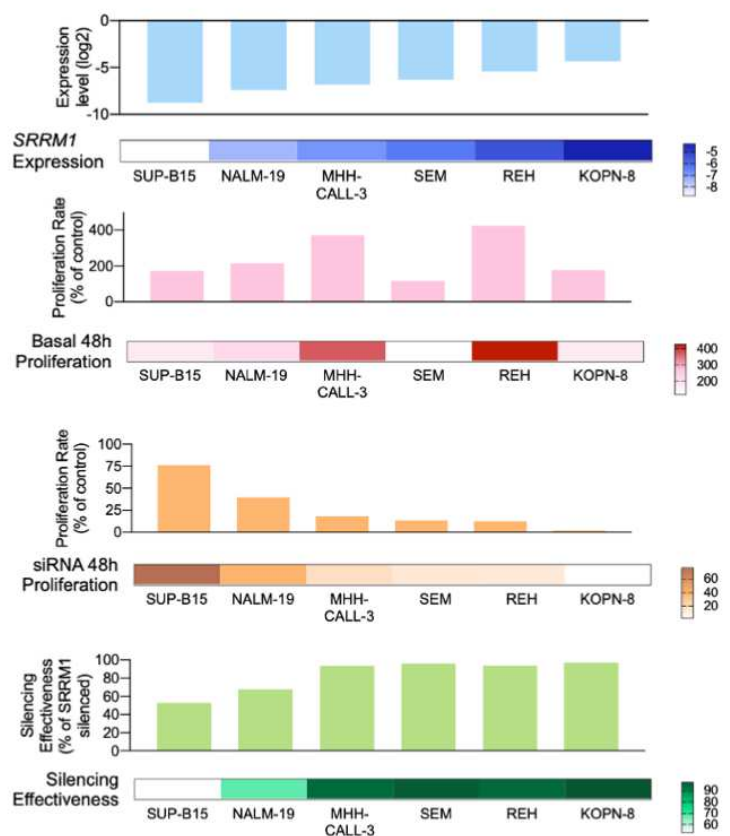

Figure 6. SRRM1 silencing in 6 human leukemia in vitro models. (a) Validation of SRRM1 silencing (y-axis) in cells ( $\mathrm{x}$-axis) by qPCR $(\mathrm{n}=3)$. (b) Proliferation rate ( $\mathrm{y}$-axis) in response to SRRM1 silencing in leukemia cell lines at different time points ( $\mathrm{x}$-axis) ( $\mathrm{n}=3$ per time point). (c) Apoptosis rate ( $\mathrm{x}$-axis) in response to SRRM1 silencing in leukemia cell lines ( $y$-axis) $(n=3)$. (d) Invasion rate $(x$-axis) in response to SRRM1 silencing in human leukemia cell lines (y-axis). Dotted lines in (c), (d) and (e) represent control condition (scramble transfected) $100 \%$. Data is presented using the mean \pm standard error of the mean. Significant differences from control conditions were indicated as * for P-value $<0.05,{ }^{* *}$ for P-value $<0.01, * * *$ for P-value $<0.001$. (e) Shows from top to bottom: comparison of SRRM1 mRNA levels, cell line proliferation in normal conditions, cell line proliferation under SRRM1 silencing, and cell line silencing effectiveness across the different human leukemia cell lines used in the functional assays by qPCR $(n=3)$. 


\section{Discussion}

In this study, we performed a multicohort age-agnostic analysis of B-ALL cases focused on the differences of risk outcome independent of the fusion background. We identified an expression pattern involving MYC targets, translational regulators, and splicing factors associated with an increased probability of relapse. Translation is tightly controlled during normal hematopoiesis (Signer et al. 2014) and is commonly deregulated in cancers, including hematological malignancies. Overexpression of $M Y C$ promotes expression of the translational machinery, increasing ribosome production and activity, leading to increased cell growth (Hodson et al. 2019). Additionally, MYC overexpression plays a role in the dysregulation of the splicing machinery during lymphomagenesis (Koh et al. 2015). Our findings suggest that alterations in RNA processing, including splicing and translation, could be involved in driving tumor progression and resistance to current therapies in BALL. This is consistent with recent work showing that aberrant splicing is directly implicated in the development of therapy resistance in B-ALL (Sisi Zheng, Elisabeth Gillespie, Ammar S. Naqvi, Katharina E. Hayer et al.; Sotillo et al. 2015)

We summarized our findings in a 37-gene signature that showed prognostic value on B-ALL patients samples. Our signature classified patients in high and low risk with high accuracy and independently of the fusion background. The same signature separated high-risk patients from normal blood and spleen samples, and B-ALL cell lines and B-cell progenitors from mature B-cells. These results provide evidence that high-risk B-ALL recapitulates an undifferentiated gene expression pattern associated with a dysregulation of RNA processing and independent of the gene fusion background. These results suggest that gene-fusions may operate mainly as initiating events, and an independent convergent mechanism defines high risk in B-ALL. Indeed, current murine and humanized models of KMT2A-r B-ALL (Lin et al. 2017) do not faithfully recapitulate the disease pathogenesis/phenotype, suggesting that KMT2A-r alone is insufficient to sustain leukemia (Prieto et al. 2016; Bueno et al. 2013). Our derived gene expression signature could complement current clinical assessment methods of B-ALL patients, mainly based on identifying gene fusions. Our signature would be beneficial as a strategy to identify patients with a high risk of relapse when no fusions are detected, or when the presented fusion is of unknown prognosis. Moreover, it is conceivable that a deeper understanding of these genes may provide further mechanistic insight into the common functional underpinnings underlying B-ALL and thus reveal potential novel therapeutic avenues. 
In line with the above notion, among the genes in our risk signature involved in RNA processing, SRRM1 provided the strongest predictive signal and was associated with splicing changes found between high and low risk patients. High-risk patients and the relapsed samples presented the highest SRRM1 expression. Moreover, SRRM1 was highly expressed in the B-ALL cell lines and B-cell progenitors, but not in mature B-cells or normal blood/spleen samples. Our analysis also showed that most of the splicing events changing between high and low-risk patients contained binding motifs for splicing factors that also presented protein-protein interactions with SRRM1, and these events occurred in genes involved in relevant cancer pathways. Interestingly, overexpression of SRRM1 has been associated with poor prognosis in prostate cancer (Jiménez-Vacas et al. 2020), and silencing of SRRM1 was shown to reduce cell proliferation through a reduction of AKT phosphorylation levels and an increased expression of PTEN, a well-known tumor suppressor (Jiménez-Vacas et al. 2020). Previous studies have also shown that SRRM1 overexpression leads to the expression of a CD44 isoform that acts as a RAS-signaling activator and induces metastatic potential in non-metastatic cells (Cheng and Sharp 2006). Furthermore, SRRM1 has been identified as part of a chromatin protein complex that drives B-cell differentiation (Ochiai et al. 2020). We showed that silencing of SRRM1 in B-ALL cell models leads to a significant decrease in proliferation and invasion rates and a significant increase in apoptosis capacity. The effect in response to SRRM1 silencing was different in all cell lines, especially in the decrease in proliferation rate. This appeared to be associated with the variable SRRM1 expression found in these cell lines rather than with the basal proliferation rate of each cell model. This would indicate that tumors with higher SRRM1 expression would depend more on SRRM1 for proliferation. In contrast, tumors with lower SRRM1 expression would rely on other proliferation mechanisms. Altogether, this leads us to propose that SRRM1 may be driving an undifferentiated or immature-cell phenotype that could sustain tumor malignancy and lead to poor prognosis in B-ALL., and that could represent a potential new therapeutic opportunity.

In conclusion, we have presented an expression signature that predicts relapse and poor outcome independently of the fusion background. This signature is associated with SRRM1 overexpression and with splicing changes potentially driven by SRRM1 interactions with other splicing factors. SRRM1 could function as a novel prognostic marker of high-risk B-ALL, and its depletion could be 
used in combination with standard therapies to achieve more effective treatments in high-risk cases.

\section{Methods}

\section{Sample selection}

All samples were obtained from various sources through controlled or public access. The series from St Jude Children's Research Hospital (SJH) (EGAS00001000246) (Andersson et al. 2015) and Lund University (LUND) (EGAS00001001795) (Lilljebjörn et al. 2016) were downloaded from the European Genome-phenome Archive (EGA), and the corresponding clinical information was obtained from the associated publications. The series from Children's Hospital of Philadelphia (CHOP) (GSE115656) was downloaded from Gene Expression Omnibus (GEO) (Black et al. 2018). Samples from TARGET (Therapeutically Applicable Research to Generate Effective Treatments) were downloaded from the TARGET data portal at National Cancer institute $(\mathrm{NIH})$ together with the associated clinical information, corresponding to dbGAP accessions phs000463 (ALL phase 1) and phs000464 (ALL phase 2). Data from patients from the Princess Maxima Center for Pediatric Oncology (PMJCI) from (Agraz-Doblas et al. 2019) was obtained from the authors. All the information related to the clinical annotations and sample extraction is described in detail in their respective publication. A detailed description of the samples selected from each project with their relevant clinical information is provided in Supp. Tables 1 and data file 1. We only used samples classified as B-cell ALL that had at least $25 \%$ of the reads mapping to the genome (GRCh38) and such that these corresponded to at least $5 \mathrm{M}$ reads. We also analyzed data from $12 \mathrm{~B}-\mathrm{ALL}$ cell lines coming from the Cancer Cell Line Encyclopedia (CCLE) (Ghandi et al. 2019), from normal blood and spleen samples from The Genotype-Tissue Expression (GTEx) project (Carithers et al. 2015), and from 16 B-cell progenitors from CHOP (GSE115656)(Black et al. 2018). All the samples were processed with the same pipeline outlined in Supp. Figure 1. FastQC (Andrews 2010) was used for quality control of the FASTQ files. FASTQ files FROM SRA for the TARGET samples were extracted using the SRAToolKit ( $v$ 2.9.0) (https://github.com/ncbi/sra-tools).

\section{Fusion detection}


We used STAR-Fusion v.1.4.0 (Haas et al. 2019) to identify gene-fusions from the RNA-seq data. The index was generated using the Gencode (v27) annotation and the GRCh38 assembly. STAR-Fusion was run for each FASTQ file using the default parameters described at https://github.com/STARFusion/STAR-Fusion/wiki/Home/. We required at least one read count supporting the fusion junction given by the field JunctRC (or JunctionReads in the latest version of the manual) in the STAR-Fusion output, one read count connecting the fusion junction (SpanRC or SpanningFrags field), 0.1 Fusion Fragments Per Million total reads (FFPM), and junction reads that cover at least 25 bases on both sides of the breakpoint (indicated as 'YES_LDAS' in the STAR-Fusion output). The fusion allele frequency (FAF) was defined as the average of the allele frequency for both partners of the fusion pair, i.e. $F A F=\left(F A F_{L}+F A F_{R}\right) / 2$, where each value $F A F_{i}$, for $i=L, R$ was defined as $F A F_{i}=F_{i} /$ $\left(F_{i}+W T_{i}\right)$, where the $F_{i}$ represents the number of reads that support the fusion breakpoint and $W T_{i}$ represents the number reads that support the wild type fragment of the gene, not present in the fusion.

\section{Fusion filtering and classification}

Fusion calls involving pseudogenes were removed from the output, as well as fusions between paralogous genes (genes with $70 \%$ or more sequence identity), as they were considered potential artifacts. Fusions between immunoglobulin or hemoglobulin genes were also discarded. Additional filters for possible false positives were applied: A promiscuity filter was used to remove fusions involving genes paired with more than one other gene within the same sample, known to be potential artifacts from the library preparation (Hsieh et al. 2017). Moreover, we removed all predicted fusions that occurred only in one project to avoid project biases. We also filtered out fusions previously detected in non-cancerous tissues or cells (Babiceanu et al. 2016), detected in normal samples from TCGA (Gao et al. 2018), or seen with STAR-fusion in RNA-seq data from normal blood cell types (Quek et al. 2016). Additionally, we only kept fusions that appeared in 5 or more patients. Fusions involving genes previously reported to have mutations or fusions in any leukemia were kept independently of these filters, but only if they appeared in 5 or more patients. Finally, the FAF was used to select the most relevant fusions (see Methods section Calculation of Fusion Allele Frequency for details). Based on the FAF distribution across all patients from the different cohorts, each fusion was required to have a median FAF greater than 0.1. The partner with the lowest allele frequency in the fusion was needed to have a median of 0.01 for the individual FAF value. 
We classified the fusions into four major groups: 1) "ALL", which indicated those already reported in any of the analyzed ALL datasets or reported previously in the databases COSMIC (Tate et al. 2019), TCGA (Gao et al. 2018), or MitelmanDB (Mitelman et al. 2020); 2) "blood", which indicated those fusions known to appear in other hematological malignancies according to the same databases; 3) "solid tumors", which indicated fusions known in other solid tumors and present in the same public databases; and 4) "novel", which indicated fusions that were not present in the public databases. Fusions were grouped and labeled according to the most recurrent partner for differential expression analysis, co-occurrence analysis, and visualization purposes. Fusion groups that were not showing a pattern of mutual exclusion with any of the other groups according to a waiting time model for mutually exclusive cancer alterations implemented by the R package TiMEx (Constantinescu et al. 2016) were grouped as "Other".

\section{Analysis of fusion disrupted domains}

The same fusion breakpoint given by the RNA-seq reads was used if this occurred inside an exon expressed in the fusion. Otherwise, the positions used were the last base of the last exon from gene 1 included in the fusion, and the first base of the first exon from gene 2 included in the fusion. We defined the breakpoints for each gene 1 and gene 2 of the identified fusions using these values. PFAM domains mapping to the proteins encoded by each fusion gene were extracted from Biomart (Smedley et al. 2009), the protein coordinates of the domain span were converted to genomic coordinates and overlapped with the fusion breakpoints of the corresponding genes to establish for each breakpoint whether the domain was kept or lost as a result of the fusion.

\section{Gene expression and functional enrichment analysis}

Transcript level quantification for the Gencode transcriptome release 27 (GRCH38.p10) (Frankish et al. 2019) was obtained in transcripts per million (TPM) units using Salmon (v 0.7.2) (Patro et al. 2017). Gene level quantification was obtained by transforming transcript TPMs to counts per gene using the tximport library function from Bioconductor (Soneson et al. 2015).

For differential expression analyses, we considered the patients with only one identified fusion (after performing all the filtering steps) and for the most frequent fusions: 87 patients for KMT2A$r, 68$ for ETV6-r, 9 for BCR-r, 36 for P2RY8-r, 14 for PAX5-r, 25 for PWLC1-r, 5 for RUNX-r, 35 for 
ST3GAL1-r, 33 for TCF3r, 33 for TTYH3-r, and 12 for ZNF384-r, plus the 133 patients with no fusions detected. We calculated the differentially expressed genes between pairs of groups. The read counts per gene were transformed to log2 counts per million (logCPM) using edgeR (Robinson et al. 2010), and genes with mean $\log C P M<0$ were filtered out. The data was normalized with the TMM method from the edgeR package. Differential expression analysis was performed with LIMMA (Ritchie et al. 2015) using the function limma.voom adjusted by SVA with the covariables of sex, project, and tissue.

Gene set enrichment analysis was performed with GSEA (Subramanian et al. 2005) for the list of hallmarks and for the biological process ontology using the pre-ranked enrichment method, sorting all the genes by the value of $-\log _{10}(p-v a l u e) \cdot \log _{2} F C$ obtained from the differential expression analysis. In the case of splicing factors and RBPs, as there is no pathway or hallmark gene set associated with them in the available databases, we built a list of genes splicing factor and/or RBP function from previous studies (Sebestyén et al. 2016; Singh et al. 2018) and run a pre-ranked GSEA with the absolute value (Data file 9) (Supp. Fig. 13).

\section{Gene selection to construct a predictive model of prognosis}

We used gene expression data from 133 TARGET patients with complete clinical information about the age of diagnosis and time to the first relapse and calculated a log2 fold-change (logFC) per gene using the normalized logCPM mean expression between patients with relapse and without relapse. Similarly, we calculated a logFC from the gene expression of 140 patients from all other cohorts (SJH, LUND, CHOP and PMJCI) comparing the ones carrying only KMT2A-r against the ones with only ETV6-r. We only considered genes with a $\operatorname{logFC}>0.5$ in both comparisons and that were included in at least one of three sets: 1 ) the GSEA hallmark as MYC targets (v1 and v2), in 2) the Gene Ontology Biological Process of translation (initiation, elongation, or termination), or 3 ) and a list of genes encoding splicing factors and RBPs (Data file 9). For every gene, we applied a cox regression survival model adjusted by age and gender, selecting only the genes with a p-value $<0.05$ (Supplementary Table 2). This produced a total of 39 overexpressed genes associated with prognosis and with our target biological functions and pathways. From these 39 genes, the expression variability between cohorts was evaluated using mean logCPM expression in each dataset and the maximum logFC between datasets. Genes with the highest variability across datasets (max logFC $>3$ ) were removed to avoid any dataset-related bias, obtaining the final 37 genes to build the predictive model. Training 
data was restricted to the 133 TARGET B-ALL patients. The model consisted in a random forest, implemented using the randomForest library in $\mathrm{R}$, with a total of 400 trees and 4 variables randomly sampled as a candidate at each split. The leave-one-out strategy was used to evaluate the prediction accuracy, while avoiding overfitting. A numeric k-score between 0-1 obtained from the prediction of the random forest model was used to classify the patients according to risk. A threshold was established on $\mathbf{0 . 7}$, according to accuracy measures, to classify the patients as high-risk (k-score >= 0.7) or low-risk (k-score < 0.7). To apply the predictive model to expression values given in terms of regularized-log (rlog) values, we used the TARGET samples as before, applying a regularized log transformation using the package DESeq2 with the rlog function (Love et al. 2014). We then built and tested the model as before, using the rlog values instead of the logCPM values.

\section{Differential splicing analysis.}

SUPPA (Alamancos et al. 2015; Trincado et al. 2018) generateEvents was used to generate alternative splicing events defined from protein-coding transcripts and covering the annotated ORFs. The relative inclusion of an event was calculated as a Percent Spliced In (PSI) value with SUPPA psiPerEvent from the transcript TPM values obtained before. A minimum total expression of the transcripts involved in the event of 1 TPM was required. Events without a defined PSI value in more than $10 \%$ of the patients across all cohorts were discarded. These included events that did not pass the transcript expression filter or that had all the transcripts involved in the event with zero expression. The remaining missing PSI values were imputed using nearest neighbor averaging with the impute.knn function in $\mathrm{R}$ from the Impute library (Hastie et al. 2020). To test the significant differential inclusion of the events in the comparisons of high against low-risk patients and in KTM2A-r against ETV6-r patients, a $\triangle \mathrm{PSI}$ was calculated as the difference of the mean PSI from each group. We discarded all events with a standard deviation (SD) across groups lower than 0.1. We applied a linear regression model with a logit transformation of the PSI to estimate the significance of the splicing changes and adjusted the $p$-value by calculating a false discovery rate (FDR). We considered significant all the changes with $|\triangle P S| \mid>0.2$ and an FDR corrected $p$-value $<0.01$. Differential splicing between B-cell precursors and GM12878 was calculated with the SUPPA diffSplice command with default options.

\section{Motif Enrichment analysis}


We searched for RBP binding motifs on the regions neighboring each splicing event with MoSEA (https://github.com/comprna/MoSEA) (Singh et al. 2018). MoSEA was run against a database of Position Frequency Matrices (PFM) and k-mers (6-mers) associated with each RBP. Enrichment was assessed by comparing a set of events differentially spliced between conditions with a set of events with no significant change between the same conditions. For each motif, MoSEA calculated a z-score from the comparison of the observed frequency observed in differentially spliced events with the distribution of frequencies in 100 control subsamples of the same size, considering the length distribution and GC content of the differentially spliced events set. We considered those motifs PFMs and 6-mers with z-score $>1.5$.

\section{Retrieving Protein-Protein Interactions}

We used the STRING database (Szklarczyk et al. 2019) to retrieve Protein-Protein Interactions with the detailed scores of the links between proteins. Only those with experimental scores different from 0 and a combined score higher than 900 were kept.

\section{Co-occurrence analysis of differentially spliced events and high risk}

An alternative exon was considered included for PSI > 0.5 and absent otherwise for each sample. For every fusion group, a matrix was built with the presence or absence of events in each patient. The co-occurrence of the events with high risk was tested with a probabilistic model of species cooccurrence implemented in the R package co-occur (Griffith et al. 2016).

\section{Functional enrichment analysis of differentially spliced genes}

Genes associated with differentially spliced events were tested for functional enrichment of Gene Ontology Biological Process terms with the R package clusterProfiler from Bioconductor (Yu et al. 2012). Benjamini-Hochberg (BH) correction was used to calculate adjusted p-values (q-values). Only ontologies with $\mathrm{p}$-value and q-value below 0.05 were selected.

\section{Leukemia cell lines selection}

SEM, MHH-CALL-3, KOPN-8, NALM-19, REH, and SUP-B15 cells were obtained from the Leibniz Institute DSMZ (\#ACC546, \#ACC339, \#ACC552, \#ACC522, \#ACC22 and \#ACC389, respectively) and cultured according to the supplier's recommendations. These cell lines were previously checked for 
mycoplasma contamination by PCR as previously reported (Uphoff and Drexler 2013). Results were expressed as a percentage with respect to scramble-transfected controls.

\section{RNA isolation, real-time qPCR, and customized qPCR dynamic array based on microfluidic technology}

Total RNA from leukemia cell lines was extracted with TRIzol ${ }^{\circledR}$ Reagent (ThermoFisher Scientific, \#15596026). Total RNA concentration and purity were assessed by Nanodrop One Microvolume UVVis Spectrophotometer (ThermoFisher Scientific). For qPCR analyses, total RNA was retrotranscribed by using random hexamer primers and the RevertAid RT Reverse Transcription Kit (ThermoFisher Scientific, \#K1691). Thermal profile and qPCR analysis to obtain absolute mRNA copy number/50 ng of sample of selected genes are reported elsewhere (Luque et al. 2015). To control for variations in the efficiency of the retrotranscription reaction, mRNA copy numbers of the different transcripts analyzed were adjusted by ACTB expression. Specific primers for human and mouse transcripts including ACTB and SRRM1 genes were specifically designed with the Primer3 software [SRRM1 (NM_001303448.1) - forward: GTAGCCCAAGAAGACGCAAA, reverse: TGGTTCTGTGACGGGGAG; ACTB (NM_001101) - forward: ACTCTTCCAGCCTTCCTTCCT, reverse: CAGTGATCTCCTTCTGCATCCT].

\section{Silencing of splicing factors by specific small interfering RNA}

Pre-designed and validated specific small interfering RNA (siRNA) oligos for knockdown of endogenous SRRM1 (\#s20018; Silencer ${ }^{\circledR}$ Select siRNAs; ThermoFisher Scientific) were used. Briefly, cells ( $n=500000$ cells/well) were transfected with $25 \mathrm{nM}$ of each siRNA individually using Lipofectamine ${ }^{\circledR} 3000$ Transfection Reagent (ThermoFisher Scientific, \# L3000075) according to the manufacturer's instructions. Silencer ${ }^{\circledR}$ Select Negative Control siRNA (ThermoFisher Scientific, \#4390843) was used as a scramble control. After $24 \mathrm{~h}$, cells were collected for validation of the transfection by qPCR and seeded for different functional assays.

\section{Proliferation rate determination}

Cell proliferation in response to SRRM1 silencing in leukemia cell lines was analyzed using alamarBlue $\mathrm{T}^{\mathrm{TM}}$ assay (Biosource International, Camarillo, CA, USA), as previously reported (FuentesFayos et al. 2020). Briefly, cells were seeded in 96 -well plates at a density of 25,000 cells/well and serum-starved for $24 \mathrm{~h}$. Then, proliferation was evaluated every $24 \mathrm{~h}$ using the FlexStation-III system 
(Molecular Devices, Sunnyvale, CA, USA) for up to $72 \mathrm{~h}$. Results were expressed as a percentage referred to scramble-transfected controls.

\section{Apoptosis measurement}

Apoptosis induction in response to SRRM1 silencing in leukemia cell lines (25,000 cells/well onto white-walled multiwell luminometer plates) was performed by using Caspase-Glo ${ }^{\circledR}$ 3/7 Assay (Promega Corporation, \#G8091) as previously reported (Fuentes-Fayos et al. 2020). Briefly, Cells were seeded in 96-well white polystyrene microplate flat bottom clearplates at a density of 25,000 cells/well and serum-starved for $24 \mathrm{~h}$. Results were expressed as a percentage referred to scrambletransfected controls.

\section{Invasion rate determination}

Invasion rate in response to SRRM1 silencing in leukemia cell lines was assessed by using the 96well cell Trans-well invasion assay (Basement Membrane-8 $\mu \mathrm{m}$, AssayGenie, \#BN01086) according to the manufacturer's protocol. The top chamber membrane was coated with the basement membrane solution. Afterward, cells were seeded in the top chamber in 0,5\% serum media. Then, $10 \%$ FBS media was placed in the bottom chamber to promote cell invasion in all experimental conditions. Then, proliferation was evaluated every 24h using the FlexStation-III system (Molecular Devices, Sunnyvale, CA, USA).

\section{Statistical analysis for the B-ALL cell line experiments}

Numerical results were evaluated for statistical differences by $\mathrm{t}$-test, multiple t-tests, and 2-way ANOVA. All statistical analyses were performed using Prism software v.9.0 (GraphPad Software, La Jolla, CA, USA). P-values $<0.05$ were considered statistically significant. Data represent median (interquartile range) or means \pm standard error of the mean (SEM). Significant difference from control conditions was indicated as * for $\mathrm{P}$-value $<0.05,{ }^{* *}$ for $\mathrm{P}$-value $<0.01, * * *$ for $\mathrm{P}$-value $<0.001$.

\section{References}

Agraz-Doblas A, Bueno C, Bashford-Rogers R, Roy A, Schneider P, Bardini M, Ballerini P, Cazzaniga G, Moreno T, Revilla C, et al. 2019. Unravelling the cellular origin and clinical prognostic 
markers of infant B-cell acute lymphoblastic leukemia using genome-wide analysis. Haematologica. http://www.ncbi.nlm.nih.gov/pubmed/30679323.

Alamancos GP, Pagés A, Trincado JL, Bellora N, Eyras E. 2015. Leveraging transcript quantification for fast computation of alternative splicing profiles. RNA 21: 1521-1531.

Andersson AK, Ma J, Wang J, Chen X, Gedman AL, Dang J, Nakitandwe J, Holmfeldt L, Parker M, Easton J, et al. 2015. The landscape of somatic mutations in infant MLL-rearranged acute lymphoblastic leukemias. Nat Genet 47.

Andrews S. 2010. FastQC: A Quality Control Tool for High Throughput Sequence Data. http://www.bioinformatics.babraham.ac.uk/projects/fastqc/.

Babiceanu M, Qin F, Xie Z, Jia Y, Lopez K, Janus N, Facemire L, Kumar S, Pang Y, Qi Y, et al. 2016. Recurrent chimeric fusion RNAs in non-cancer tissues and cells. Nucleic Acids Res $4 \mathbf{4}$. http://www.ncbi.nlm.nih.gov/pubmed/26837576.

Black KL, Naqvi AS, Asnani M, Hayer KE, Yang SY, Gillespie E, Bagashev A, Pillai V, Tasian SK, Gazzara MR, et al. 2018. Aberrant splicing in B-cell acute lymphoblastic leukemia. Nucleic Acids Res 46: 11357-11369. http://www.ncbi.nlm.nih.gov/pubmed/30357359.

Blencowe BJ, Issner R, Nickerson JA, Sharp PA. 1998. A coactivator of pre-mRNA splicing. Genes Dev 12: 996-1009. http://www.ncbi.nlm.nih.gov/pubmed/9531537.

Boer JM, den Boer ML. 2017. BCR-ABL1-like acute lymphoblastic leukaemia: From bench to bedside. Eur J Cancer 82: 203-218. http://www.ncbi.nlm.nih.gov/pubmed/28709134.

Bueno C, Ayllón V, Montes R, Navarro-Montero O, Ramos-Mejia V, Real PJ, Romero-Moya D, Araúzo-Bravo MJ, Menendez P. 2013. FLT3 activation cooperates with MLL-AF4 fusion protein to abrogate the hematopoietic specification of human ESCs. Blood 121: 3867-78, S1-3. http://www.ncbi.nlm.nih.gov/pubmed/23479570.

Bueno C, Montes R, Catalina P, Rodríguez R, Menendez P. 2011. Insights into the cellular origin and etiology of the infant pro-B acute lymphoblastic leukemia with MLL-AF4 rearrangement. Leukemia 25. http://www.ncbi.nlm.nih.gov/pubmed/21135858.

Bueno C, Montes R, Melen GJ, Ramos-Mejia V, Real PJ, Ayllón V, Sanchez L, Ligero G, GutierrezAranda I, Fernández AF, et al. 2012. A human ESC model for MLL-AF4 leukemic fusion gene reveals an impaired early hematopoietic-endothelial specification. Cell Res 22: 986-1002. http://www.ncbi.nlm.nih.gov/pubmed/22212479.

Bursen A, Schwabe K, Rüster B, Henschler R, Ruthardt M, Dingermann T, Marschalek R. 2010. The AF4.MLL fusion protein is capable of inducing ALL in mice without requirement of MLL·AF4. 
Blood 115: 3570-3579.

Carithers LJ, Ardlie K, Barcus M, Branton PA, Britton A, Buia SA, Compton CC, DeLuca DS, PeterDemchok J, Gelfand ET, et al. 2015. A Novel Approach to High-Quality Postmortem Tissue Procurement: The GTEx Project. Biopreserv Biobank 13: 311-9.

http://www.ncbi.nIm.nih.gov/pubmed/26484571.

Cheng C, Sharp PA. 2006. Regulation of CD44 alternative splicing by SRm160 and its potential role in tumor cell invasion. Mol Cell Biol 26: 362-70.

http://www.ncbi.nlm.nih.gov/pubmed/16354706.

Constantinescu S, Szczurek E, Mohammadi P, Rahnenführer J, Beerenwinkel N. 2016. TiMEx: a waiting time model for mutually exclusive cancer alterations. Bioinformatics 32: 968-75. http://www.ncbi.nlm.nih.gov/pubmed/26163509.

Fischer U, Forster M, Rinaldi A, Risch T, Sungalee S, Warnatz H-J, Bornhauser B, Gombert M, Kratsch C, Stütz AM, et al. 2015. Genomics and drug profiling of fatal TCF3-HLF-positive acute lymphoblastic leukemia identifies recurrent mutation patterns and therapeutic options. Nat Genet 47: 1020-1029. http://www.ncbi.nlm.nih.gov/pubmed/26214592.

Frankish A, Diekhans M, Ferreira A-M, Johnson R, Jungreis I, Loveland J, Mudge JM, Sisu C, Wright J, Armstrong J, et al. 2019. GENCODE reference annotation for the human and mouse genomes. Nucleic Acids Res 47: D766-D773.

http://www.ncbi.nlm.nih.gov/pubmed/30357393.

Fuentes-Fayos AC, Vázquez-Borrego MC, Jiménez-Vacas JM, Bejarano L, Pedraza-Arévalo S, L.López F, Blanco-Acevedo C, Sánchez-Sánchez R, Reyes O, Ventura S, et al. 2020. Splicing machinery dysregulation drives glioblastoma development/aggressiveness: oncogenic role of SRSF3. Brain 143: 3273-3293.

https://academic.oup.com/brain/article/143/11/3273/5952721.

Gao Q, Liang W-W, Foltz SM, Mutharasu G, Jayasinghe RG, Cao S, Liao W-W, Reynolds SM, Wyczalkowski MA, Yao L, et al. 2018. Driver Fusions and Their Implications in the Development and Treatment of Human Cancers. Cell Rep 23: 227-238.e3.

Ghandi M, Huang FW, Jané-Valbuena J, Kryukov G V, Lo CC, McDonald ER, Barretina J, Gelfand ET, Bielski CM, Li H, et al. 2019. Next-generation characterization of the Cancer Cell Line Encyclopedia. Nature 569: 503-508. http://www.ncbi.nlm.nih.gov/pubmed/31068700.

Griffith DM, Veech JA, Marsh CJ. 2016. cooccur : Probabilistic Species Co-Occurrence Analysis in R. J Stat Softw 69. http://www.jstatsoft.org/v69/c02/. 
Gröbner SN, Worst BC, Weischenfeldt J, Buchhalter I, Kleinheinz K, Rudneva VA, Johann PD, Balasubramanian GP, Segura-Wang M, Brabetz S, et al. 2018. The landscape of genomic alterations across childhood cancers. Nature 555: 321-327.

Haas BJ, Dobin A, Li B, Stransky N, Pochet N, Regev A. 2019. Accuracy assessment of fusion transcript detection via read-mapping and de novo fusion transcript assembly-based methods. Genome Biol 20: 213. http://www.ncbi.nlm.nih.gov/pubmed/31639029.

Hastie T, Tibshirani R, Narasimhan B, Chu G. 2020. impute: impute: Imputation for microarray data.

Hodson DJ, Screen M, Turner M. 2019. RNA-binding proteins in hematopoiesis and hematological malignancy. Blood 133: 2365-2373. http://www.ncbi.nlm.nih.gov/pubmed/30967369.

Hsieh G, Bierman R, Szabo L, Lee AG, Freeman DE, Watson N, Sweet-Cordero EA, Salzman J. 2017. Statistical algorithms improve accuracy of gene fusion detection. Nucleic Acids Res 45. http://www.ncbi.nlm.nih.gov/pubmed/28541529.

Jeha S, Choi J, Roberts KG, Pei D, Coustan-Smith E, Inaba H, Rubnitz JE, Ribeiro RC, Gruber TA, Raimondi SC, et al. 2021. Clinical Significance of Novel Subtypes of Acute Lymphoblastic Leukemia in the Context of Minimal Residual Disease-Directed Therapy. Blood Cancer Discov 2: 326-337. http://bloodcancerdiscov.aacrjournals.org/lookup/doi/10.1158/2643-3230.BCD20-0229.

Jiménez-Vacas JM, Herrero-Aguayo V, Montero-Hidalgo AJ, Gómez-Gómez E, Fuentes-Fayos AC, León-González AJ, Sáez-Martínez P, Alors-Pérez E, Pedraza-Arévalo S, González-Serrano T, et al. 2020. Dysregulation of the splicing machinery is directly associated to aggressiveness of prostate cancer. EBioMedicine 51: 102547. http://www.ncbi.nlm.nih.gov/pubmed/31902674. Koh CM, Bezzi M, Low DHP, Ang WX, Teo SX, Gay FPH, Al-Haddawi M, Tan SY, Osato M, Sabò A, et al. 2015. MYC regulates the core pre-mRNA splicing machinery as an essential step in lymphomagenesis. Nature 523: 96-100.

Krivtsov A V, Armstrong SA. 2007. MLL translocations, histone modifications and leukaemia stemcell development. Nat Rev Cancer 7. http://www.ncbi.nlm.nih.gov/pubmed/17957188.

Lilljebjörn H, Henningsson R, Hyrenius-Wittsten A, Olsson L, Orsmark-Pietras C, von Palffy S, Askmyr M, Rissler M, Schrappe M, Cario G, et al. 2016. Identification of ETV6-RUNX1-like and DUX4-rearranged subtypes in paediatric B-cell precursor acute lymphoblastic leukaemia. Nat Commun 7: 11790. http://www.ncbi.nlm.nih.gov/pubmed/27265895.

Lin S, Luo RT, Shrestha M, Thirman MJ, Mulloy JC. 2017. The full transforming capacity of MLL-Af4 
is interlinked with lymphoid lineage commitment. Blood 130: 903-907.

http://www.ncbi.nlm.nih.gov/pubmed/28637661.

Love MI, Huber W, Anders S. 2014. Moderated estimation of fold change and dispersion for RNAseq data with DESeq2. Genome Biol 15: 550.

http://genomebiology.biomedcentral.com/articles/10.1186/s13059-014-0550-8.

Luque RM, Ibáñez-Costa A, Neto LV, Taboada GF, Hormaechea-Agulla D, Kasuki L, Venegas-

Moreno E, Moreno-Carazo A, Gálvez MÁ, Soto-Moreno A, et al. 2015. Truncated somatostatin receptor variant sst5TMD4 confers aggressive features (proliferation, invasion and reduced octreotide response) to somatotropinomas. Cancer Lett 359: 299-306.

https://linkinghub.elsevier.com/retrieve/pii/S0304383515000713.

Ma X, Edmonson M, Yergeau D, Muzny DM, Hampton OA, Rusch M, Song G, Easton J, Harvey RC, Wheeler DA, et al. 2015. Rise and fall of subclones from diagnosis to relapse in pediatric Bacute lymphoblastic leukaemia. Nat Commun 6: 6604.

http://www.ncbi.nlm.nih.gov/pubmed/25790293.

Meyer C, Burmeister T, Gröger D, Tsaur G, Fechina L, Renneville A, Sutton R, Venn NC, Emerenciano M, Pombo-de-Oliveira MS, et al. 2018. The MLL recombinome of acute leukemias in 2017. Leukemia 32: 273-284. http://www.ncbi.nlm.nih.gov/pubmed/28701730.

Mitelman F, Johansson B, Mertens F. 2020. Mitelman Database of Chromosome Aberrations and Gene Fusions in Cancer. https://mitelmandatabase.isb-cgc.org.

Montes R, Ayllón V, Gutierrez-Aranda I, Prat I, Hernández-Lamas MC, Ponce L, Bresolin S, Te Kronnie G, Greaves M, Bueno C, et al. 2011. Enforced expression of MLL-AF4 fusion in cord blood CD34+ cells enhances the hematopoietic repopulating cell function and clonogenic potential but is not sufficient to initiate leukemia. Blood 117: 4746-58.

Ning J, Zhao Y, Ye Y, Yu J. 2019. Opposing roles and potential antagonistic mechanism between TGF- $\beta$ and BMP pathways: Implications for cancer progression. EBioMedicine 41: 702-710. http://www.ncbi.nIm.nih.gov/pubmed/30808576.

Ochiai K, Yamaoka M, Swaminathan A, Shima H, Hiura H, Matsumoto M, Kurotaki D, Nakabayashi J, Funayama R, Nakayama K, et al. 2020. Chromatin Protein PC4 Orchestrates B Cell Differentiation by Collaborating with IKAROS and IRF4. Cell Rep 33: 108517. https://linkinghub.elsevier.com/retrieve/pii/S2211124720315060.

Patro R, Duggal G, Love MI, Irizarry RA, Kingsford C. 2017. Salmon provides fast and bias-aware quantification of transcript expression. Nat Methods 14: 417-419. 
http://www.ncbi.nlm.nih.gov/pubmed/28263959.

Pikman Y, Stegmaier K. 2018. Targeted therapy for fusion-driven high-risk acute leukemia. Blood 132: 1241-1247. http://www.ncbi.nIm.nih.gov/pubmed/30049809.

Prieto C, Stam RW, Agraz-Doblas A, Ballerini P, Camos M, Castaño J, Marschalek R, Bursen A, Varela I, Bueno C, et al. 2016. Activated KRAS Cooperates with MLL-AF4 to Promote Extramedullary Engraftment and Migration of Cord Blood CD34+ HSPC But Is Insufficient to Initiate Leukemia. Cancer Res 76: 2478-89. http://www.ncbi.nlm.nih.gov/pubmed/26837759.

Pui C-H, Evans WE. 2013. A 50-year journey to cure childhood acute lymphoblastic leukemia. Semin Hematol 50: 185-96.

Quek L, Otto GW, Garnett C, Lhermitte L, Karamitros D, Stoilova B, Lau I-J, Doondeea J, Usukhbayar B, Kennedy A, et al. 2016. Genetically distinct leukemic stem cells in human CD34- acute myeloid leukemia are arrested at a hemopoietic precursor-like stage. J Exp Med 213. http://www.ncbi.nlm.nih.gov/pubmed/27377587.

Ritchie ME, Phipson B, Wu D, Hu Y, Law CW, Shi W, Smyth GK. 2015. limma powers differential expression analyses for RNA-sequencing and microarray studies. Nucleic Acids Res 43. http://www.ncbi.nlm.nih.gov/pubmed/25605792.

Robinson MD, McCarthy DJ, Smyth GK. 2010. edgeR: a Bioconductor package for differential expression analysis of digital gene expression data. Bioinformatics 26: 139-40.

Sebestyén E, Singh B, Miñana B, Pagès A, Mateo F, Pujana MA, Valcárcel J, Eyras E. 2016. Largescale analysis of genome and transcriptome alterations in multiple tumors unveils novel cancer-relevant splicing networks. Genome Res 26.

Signer RAJ, Magee JA, Salic A, Morrison SJ. 2014. Haematopoietic stem cells require a highly regulated protein synthesis rate. Nature 509: 49-54. http://www.ncbi.nlm.nih.gov/pubmed/24670665.

Singh B, Trincado JL, Tatlow PJ, Piccolo SR, Eyras E. 2018. Genome Sequencing and RNA-Motif Analysis Reveal Novel Damaging Noncoding Mutations in Human Tumors. Mol Cancer Res 16: 1112-1124. http://www.ncbi.nlm.nih.gov/pubmed/29592900.

Sisi Zheng, Elisabeth Gillespie, Ammar S. Naqvi, Katharina E. Hayer Z, Ang, Manuel Torres-Diz, Mathieu Quesnel-Vallières, David A. Hottman A, Bagashev, John Chukinas, Carolin Schmidt, Mukta Asnani, Rawan Shraim, Deanne M. Taylor, Susan R. Rheingold, Maureen M. O’Brien NS, Kristen W. Lynch, Marco Ruella, Yoseph Barash, Sarah K. Tasian and A, ThomasTikhonenko. Modulation of CD22 protein expression in childhood leukemia by pervasive 
splicing aberrations: implications for CD22-directed immunotherapies. Blood Cancer Discov. Smedley D, Haider S, Ballester B, Holland R, London D, Thorisson G, Kasprzyk A. 2009. BioMart-biological queries made easy. BMC Genomics 10: 22. http://www.ncbi.nlm.nih.gov/pubmed/19144180.

Soneson C, Love MI, Robinson MD. 2015. Differential analyses for RNA-seq: transcript-level estimates improve gene-level inferences. F1000Research 4: 1521. http://www.ncbi.nlm.nih.gov/pubmed/26925227.

Sotillo E, Barrett DM, Black KL, Bagashev A, Oldridge D, Wu G, Sussman R, Lanauze C, Ruella M, Gazzara MR, et al. 2015. Convergence of acquired mutations and alternative splicing of CD19 enables resistance to CART-19 immunotherapy. Cancer Discov 5: 1282-1295.

Subramanian A, Tamayo P, Mootha VK, Mukherjee S, Ebert BL. 2005. Gene set enrichment analysis : A knowledge-based approach for interpreting genome-wide.

Szklarczyk D, Gable AL, Lyon D, Junge A, Wyder S, Huerta-Cepas J, Simonovic M, Doncheva NT, Morris JH, Bork P, et al. 2019. STRING v11: protein-protein association networks with increased coverage, supporting functional discovery in genome-wide experimental datasets. Nucleic Acids Res 47: D607-D613. https://academic.oup.com/nar/article/47/D1/D607/5198476.

Tasian SK, Loh ML, Hunger SP. 2017. Philadelphia chromosome-like acute lymphoblastic leukemia. Blood 130: 2064-2072. http://www.ncbi.nlm.nih.gov/pubmed/28972016.

Tate JG, Bamford S, Jubb HC, Sondka Z, Beare DM, Bindal N, Boutselakis H, Cole CG, Creatore C, Dawson E, et al. 2019. COSMIC: the Catalogue Of Somatic Mutations In Cancer. Nucleic Acids Res 47: D941-D947. http://www.ncbi.nlm.nih.gov/pubmed/30371878.

Trincado JL, Entizne JC, Hysenaj G, Singh B, Skalic M, Elliott DJ, Eyras E. 2018. SUPPA2: Fast, accurate, and uncertainty-aware differential splicing analysis across multiple conditions. Genome Biol 19.

Uphoff CC, Drexler HG. 2013. Detection of mycoplasma contaminations. Methods Mol Biol 946: 113. http://www.ncbi.nlm.nih.gov/pubmed/23179822.

Vaysse C, Philippe C, Martineau Y, Quelen C, Hieblot C, Renaud C, Nicaise Y, Desquesnes A, Pannese M, Filleron T, et al. 2015. Key contribution of elF4H-mediated translational control in tumor promotion. Oncotarget 6: 39924-40. http://www.ncbi.nlm.nih.gov/pubmed/26498689.

Wang Y, Wu N, Liu D, Jin Y. 2017. Recurrent Fusion Genes in Leukemia: An Attractive Target for 
Diagnosis and Treatment. Curr Genomics 18: 378-384.

http://www.ncbi.nlm.nih.gov/pubmed/29081694 (Accessed October 19, 2018).

Yu G, Wang L-G, Han Y, He Q-Y. 2012. clusterProfiler: an R package for comparing biological themes among gene clusters. OMICS 16: 284-7.

http://www.ncbi.nIm.nih.gov/pubmed/22455463.

\section{Description of data files}

Data file 1. Table with detailed clinical information for all patients obtained from the original sources. Sample_ID is the code used to identify the sample. Patient_ID is the code used to identify the patient. Project indicates the sample cohort of origin. Type indicates the type of leukemia. Tissue indicates the origin of the sample. Blasts indicates the proportion of blasts in the sample. DX_RL indicates the time when the sample was obtained at diagnosis (DX) or after relapse (RL). Gender indicates female (F) or male (M) for each patient. Age indicates the age of the patient in months. Fusions indicates the fusion recorded in the clinical information. Cell.of.Origin indicates if the samples where obtained from B-cells or B-cells precursors. First.event indicates if the patient had a relapse or not. Event.Free.survival.time.in.days is the time in days where the first event appeared. Vital.Status indicates if the patient is dead or not. Overall.Survival.Time.in.Days is the time until the dead of the patient or the last time of follow up.

Data file 2. Table with all fusions detected. Columns indicate sample code (Sample), patient ID (ID), cohort, source of the fusion calling (Star-fusion, Clinical information, or both), cancer type in which the fusion has been observed before (Cancer_category), fusion group in which the fusion has been assigned (Group), number of reads supporting the fusion junction (JunctRC), number of spanning reads supporting the fusion (SpanRC), expression of the fusion in Fusion Fragments per Million (FFPM), Ensembl gene identifier of the first fusion gene (ENG1), breakpoint coordinates of the first fusion gene (BP1), Ensembl gene identifier of the second fusion gene (ENSG2), breakpoint of the second fusion gene (BP2), type of chromosomal rearrangement (annotation), FAF of the first fusion gene (FAF_gene1), FAF of the second fusion gene (FAF_gene2), and combined FAF (FAF). 
Data file 3. Tables with all differential expression analysis between fusion groups. The comparisons and labels correspond to those shown in the supplementary figure 9. GeneID column indicate the gene name. LogFC is the log2-fold-change between the two conditions. AveExpr is the average log2expression for the gene over all samples. $\mathrm{T}$ is the moderated t-statistic. P.Value is the raw $\mathrm{p}$-value. Adj.P.Val is the adjusted p-value using the BH method. B is the log-odds that the gene is differentially expressed.

Data file 4. Differential expression analysis between KMT2A-r and ETV6-r patients. GeneID column indicates the gene name. LogFC is the log2-fold-change between the two conditions. AveExpr is the average log2-expression for the gene over all samples. $T$ is the moderated t-statistic. P.Value is the raw $p$-value. Adj.P.Val is the adjusted $p$-value using the $\mathrm{BH}$ method. $\mathrm{B}$ is the log-odds that the gene is differentially expressed.

Data file 5. Signature scores for all the patients and cell lines studied. Column Sample indicates the sample code. Column None indicates the score associated to not having a relapse. Column Relapse indicates the score associated to not having a relapse. Column Sample_type indicates if the origin of the sample is a patient at diagnostic stage (Diagnostic), a patient at relapse stage (Relapse), a cancer cell line (CCLE) or a normal sample (GM12878 cell line, B-cell progenitors).

Data file 6. Differential splicing events between high and low risk patients. Columns indicate the event ID, dPSI, p-value and FDR adjusted p-value as calculated with the linear model (see Methods), HGNC gene symbol, transcripts supporting the inclusion of the event (Alt 1), transcripts supporting the exclusion of the event (Alt 2), column indication whether any of Alt 1 or Alt 2 transcripts are annotated as coding (transcript_type), cancer hallmarks associated to the differentially spliced genes (hallmarks), fusions in which the event shows cooccurrence with high risk (high_risk_cooccur) and presence of motifs for SRRM1 interacting RBPs(SRRM1_RBPs_motifs).

Data file 7. Differential splicing events between $K M T 2 A-r$ and ETV6-r patients. Columns indicate the event IDs, dPSI, p-value and FDR adjusted p-value as calculated with the linear model (see Methods).

Data table 8. Differential splicing events between B-cell precursors and GM12878 cells. Columns indicate the event ID, dPSI and p-value as provided by SUPPA. Events for which the method could not retrieve a dPSI value were omitted (see Methods). 
Data file 9. Manually curated list of RBPs and splicing factors used for the analysis. 


\section{Supplementary Files}

This is a list of supplementary files associated with this preprint. Click to download.

- figuressupplementary17.pdf

- datafile1clinicalinfo.xIsx

- datafile2fusions.txt

- datafile3DEall.xIsx

- datafile4DEkmt2avsetv6.txt

- datafile5scores.xlsx

- datafile6splicingsummarytable.txt

- datafile7sigpsilmprojecttpm1 kmt2avsetv6.txt

- datafile8splicingBprecursorsvsGM1878.txt

- datafile9genesetsfrbp.xlsx 